\title{
Epitope-based vaccine design yields fusion peptide-directed antibodies that neutralize diverse strains of HIV-1
}

\author{
Kai Xu1,9, Priyamvada Acharya1,2,9, Rui Kong ${ }^{1,9}$, Cheng Cheng 1,9, Gwo-Yu Chuang1, Kevin Liu (1) , \\ Mark K. Louder (D), Sijy O'Dell', Reda Rawi', Mallika Sastry', Chen-Hsiang Shen', Baoshan Zhang', \\ Tongqing Zhou', Mangaiarkarasi Asokan', Robert T. Bailer', Michael Chambers', Xuejun Chen', \\ Chang W. Choi', Venkata P. Dandey², Nicole A. Doria-Rose', Aliaksandr Druz', Edward T. Eng², \\ S. Katie Farney1, Kathryn E. Foulds', Hui Geng'1, Ivelin S. Georgiev³, Jason Gorman', Kurt R. Hill', \\ Alexander J. Jafari', Young D. Kwon', Yen-Ting Lai®1, Thomas Lemmin', Krisha McKee', \\ Tiffany Y. Ohr', Li Ou', Dongjun Peng1, Ariana P. Rowshan', Zizhang Sheng ${ }^{5,6}$, John-Paul Todd', \\ Yaroslav Tsybovsky, Elise G. Viox', Yiran Wang ${ }^{1}$, Hui Wei ${ }^{2}$, Yongping Yang ${ }^{1}$, Amy F. Zhou', \\ Rui Chen ${ }^{8}$, Lu Yang ${ }^{8}$, Diana G. Scorpio', Adrian B. McDermott', Lawrence Shapiro ${ }^{1,5,6}$, \\ Bridget Carragher ${ }^{2}$, Clinton S. Potter ${ }^{2}$, John R. Mascola ${ }^{1 \star}$ and Peter D. Kwong ${ }^{1,5 \star}$
}

A central goal of HIV-1 vaccine research is the elicitation of antibodies capable of neutralizing diverse primary isolates of HIV-1. Here we show that focusing the immune response to exposed $\mathbf{N}$-terminal residues of the fusion peptide, a critical component of the viral entry machinery and the epitope of antibodies elicited by HIV-1 infection, through immunization with fusion peptidecoupled carriers and prefusion stabilized envelope trimers, induces cross-clade neutralizing responses. In mice, these immunogens elicited monoclonal antibodies capable of neutralizing up to $31 \%$ of a cross-clade panel of 208 HIV-1 strains. Crystal and cryoelectron microscopy structures of these antibodies revealed fusion peptide conformational diversity as a molecular explanation for the cross-clade neutralization. Immunization of guinea pigs and rhesus macaques induced similarly broad fusion peptide-directed neutralizing responses, suggesting translatability. The $\mathbf{N}$ terminus of the HIV-1 fusion peptide is thus a promising target of vaccine efforts aimed at eliciting broadly neutralizing antibodies.

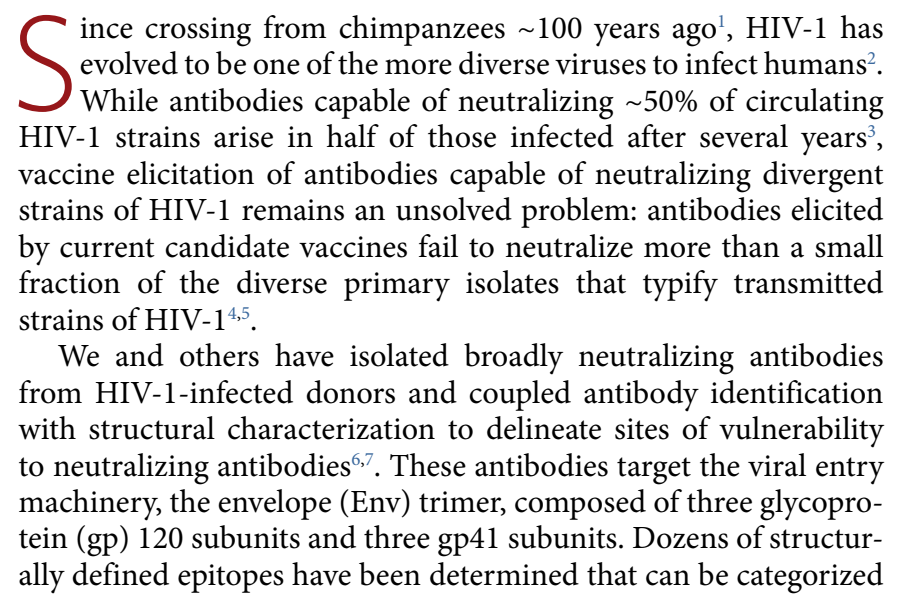

into a handful of Env regions. The majority of identified neutralizing antibodies have characteristics that may make them difficult to elicit by vaccination, including those to the CD4-binding site ${ }^{8,9}$, where extensive somatic hypermutation (SHM) appears to be required ${ }^{10-12}$; those to a quaternary site at the trimer apex ${ }^{13-16}$, where unusual recombination appears to be required ${ }^{13,14,17,18}$; those to a glycan-V3 supersite ${ }^{19-21}$, where recognition of $N$-linked glycan appears to be required ${ }^{20-22}$; and those to the membrane-proximal external region $^{23-26}$, where co-recognition of membrane ${ }^{27-29}$ and immune tolerance appear to be required ${ }^{30}$

Recently, we identified an antibody, N123-VRC34.01 ${ }^{31}$, named for donor (N123), lineage (VRC34), and clone number (01), and hereafter referenced without donor prefix. VRC34.01 targets primarily the conserved N-terminal region of the HIV-1 fusion peptide (FP), a critical component of viral entry machinery ${ }^{32}$. FP is composed of 15-20 hydrophobic amino acids at the $\mathrm{N}$ terminus of

'Vaccine Research Center, National Institute of Allergy and Infectious Diseases, National Institutes of Health, Bethesda, MD, USA. ${ }^{2}$ National Resource for Automated Molecular Microscopy, Simons Electron Microscopy Center, New York Structural Biology Center, New York, NY, USA. ${ }^{3}$ Vanderbilt Vaccine Center, Department of Pathology, Microbiology, and Immunology, Vanderbilt University Medical Center, and Department of Electrical Engineering and Computer Science, Vanderbilt University, Nashville, TN, USA. ${ }^{4}$ Department of Pharmaceutical Chemistry, University of California San Francisco, San Francisco, CA, USA. '5 Department of Biochemistry and Molecular Biophysics, Columbia University, New York, NY, USA. ${ }^{6}$ Department of Systems Biology, Columbia University, New York, NY, USA. 'Electron Microscopy Laboratory, Cancer Research Technology Program, Leidos Biomedical Research, Inc., Frederick National Laboratory for Cancer Research, Frederick, MD, USA. ${ }^{8}$ GenScript USA, Piscataway, NJ, USA. ${ }^{9}$ These authors contributed equally: Kai Xu, Priyamvada Acharya, Rui Kong, Cheng Cheng. *e-mail: jmascola@nih.gov; pdkwong@nih.gov 
the gp41 subunit of HIV Env and had been thought to be poorly immunogenic: hidden from the immune system in the native prefusion state of Env and buried in the cell membrane after Env rearranges into the postfusion state. The VRC34.01 antibody, however, revealed the $\mathrm{N}$-terminal half of $\mathrm{FP}$ to be a site of neutralization vulnerability. VRC34.01 directs the majority of its binding energy to eight $\mathrm{N}$-terminal residues of $\mathrm{FP}$, with the rest coming from interactions with Env including glycan $\mathrm{N} 88^{31}$. The ability to neutralize HIV-1 through recognition of a linear peptide that is both conserved in sequence and exposed in the prefusion, closed conformation of Env, suggested that the site of vulnerability defined by VRC34.01 might be amenable to epitope-based approaches to vaccine design. In this study, we describe an antibody-to-vaccine development process. Beginning with the epitope of VRC34.01, we engineered immunogens with antigenic specificity for FP-directed antibodies, immunized C57BL/6 mice, and analyzed the resultant antibodies. Based on these analyses, we devised second-generation immunization regimens that generated improved neutralizing monoclonal antibodies. We extracted insights from the murine immunizations and applied these to immunize guinea pigs and rhesus macaques. While breadth, potency, and consistency need to be improved, overall, these vaccine studies demonstrated the ability, in standard vaccine-test species, to induce serum responses capable of neutralizing a substantial fraction of primary isolate strains representative of the global diversity of HIV-1.

Fusion peptide-based immunogens. The eight $\mathrm{N}$-terminal residues of FP were chosen as a vaccine target, based on their recognition by antibody VRC34.01 in co-crystal structures and on molecular dynamics simulations, which showed these residues to be exposed and flexible in conformation ${ }^{31}$. To obtain immunogens capable of eliciting FP-directed antibodies, we used structure-based design to engineer FP-containing immunogens and assessed their antigenic specificity against a panel of antibodies encompassing both broadly neutralizing antibodies and poorly or non-neutralizing antibodies, with an emphasis on antibodies reported to engage FP as part of their recognized epitope such as ACS202 $2^{33}$ and PGT151 $1^{34}$ (Fig. 1a). We used an antigenicity score derived from the binding affinity of epitope-specific neutralizing and weakly or non-neutralizing antibodies (see Methods) to estimate the epitope-specific suitability of each immunogen (Supplementary Fig. 1a). We produced epitope scaffolds that incorporated the eight $\mathrm{N}$-terminal amino acids of FP and, in some cases, included sites of $N$-linked glycosylation, which we positioned analogously to FP and glycan N88 in the VRC34.01 epitope. We characterized two scaffolds, which were trimeric and tetrameric, from Protein Data Bank (PDB) $3 \mathrm{HSH}^{35}$ and $1 \mathrm{SLF}^{36}$, respectively, and also assessed epitope scaffolds engineered from PDB $1 \mathrm{M}^{2} \mathrm{~T}^{37}$ and from PDB $1 \mathrm{Y} 12^{38}$, which we previously described ${ }^{31}$ (Supplementary Fig. 1b-g); the oligomeric scaffolds generally showed stronger binding to the FP-directed antibodies.

We also created a FP-carrier protein conjugate, by coupling the eight $\mathrm{N}$-terminal residues of $\mathrm{FP}$ through an appended $\mathrm{C}$-terminal cysteine using a bifunctional reagent to covalently link to lysine residues in keyhole limpet hemocyanin (KLH), a carrier protein often used to improve immunogenicity ${ }^{39}$. The resultant FP8-KLH was robustly recognized by FP-directed antibodies and stable to extremes of temperature and osmolality, and at $\mathrm{pH} 10.0$, but not at $\mathrm{pH} 3.5$; and negative-stain electron microscopy (EM) revealed that FP-conjugated KLH retained the barrel shape of KLH (Supplementary Fig. 1b,c,h,i). When assessed with the FP-antigenicity score (Fig. 1a), FP8-KLH was superior to the FP-epitope scaffolds and similar to stabilized Env trimers, such as the SOSIP.664 ${ }^{40}$ or DS-SOSIP ${ }^{41}$ trimers from the clade A strain BG505.

Immunogens with high FP-antigenic specificity induce FP-directed neutralizing responses. To assess the ability of the first-generation epitope-based vaccine immunogens to elicit neutralizing responses, we tested immunization regimens using the two immunogens with the highest FP-antigenicity scores, FP8-KLH and stabilized Env trimer. In an initial experiment, 4 C57BL/6 mice each received $50 \mu \mathrm{g}$ of BG505 SOSIP Env trimer and were boosted with $25 \mu \mathrm{g}$ of FP8-KLH at day 14 (Fig. 1b). After a second boost at day 28, strong FP responses in enzyme-linked immunosorbent assay (ELISA) were observed at day 35. After a final boost at day 49 , we tested day-52 serum for neutralization of the Env pseudovirus BG505 and for neutralization of a BG505 Env variant missing glycans at positions 88 or 611 ( $\Delta 88$ or $\Delta 611$, respectively), as these Env variants are more sensitive to FP-directed antibodies ${ }^{31}$. While serum neutralization of wild-type BG505 generally did not pass our $\mathrm{ID}_{50}$ (reciprocal plasma or serum dilutions causing a $50 \%$ reduction) threshold for murine neutralization (at least 1:40 and at least twofold above background), we did observe unambiguous serum neutralization for the $\Delta 88+611$ glycan-deleted variant of BG505 by all 4 of the mice (Fig. 1c and Supplementary Fig. 2a).

In a subsequent experiment, 3 mice each received $25 \mu \mathrm{g}$ of FP-KLH, followed by boosts at day 14 and day 35 (Fig. 1d). Serum ELISAs revealed Env trimer recognition in mouse 2586 at days 21 and 38; this also appeared in a second mouse after the final boost. We tested day-38 serum for neutralization and observed neutralization for the $\Delta 88+611$ glycan-deleted variant of BG505 by two of the sera (Fig. 1e and Supplementary Fig. 2a).

First generation FP-directed antibodies neutralize up to $\sim 10 \%$ of HIV-1 strains. To provide insight into the antibodies elicited by FP-containing immunogens, we selected B cell hybridomas capable of binding both BG505 SOSIP trimer and FP-1M6T scaffold, using B cells from mouse 1868 (immunized with Env trimer and FP-KLH) and mouse 2586 (immunized with FP-KLH only). Sequences of seven hybridomas from mouse 1868 and five hybridomas from mouse 2586 revealed seven vaccine-elicited (v) antibody lineages (vFP1vFP7), which could be segregated into three classes defined by B cell ontogeny and structural mode of recognition ${ }^{6}$ and which we named vFP1, vFP5, and vFP6, after the first identified member of each class (Supplementary Fig. 3a,b and Supplementary Tables 1 and 2).

We tested neutralization of the $12 \mathrm{vFP}$ antibodies on a panel of wild-type and glycan-deleted HIV-1 variants. Clear neutralization of wild-type HIV-1 strains was observed with only a few of the vFP1-class antibodies (vFP1.01, vFP7.04, and vFP7.05). We assessed the three best vFP1-class antibodies along with antibody vFP5.01 on a well-characterized panel of 208 Env pseudoviruses, encompassing diverse strains of primary isolates from all of the major clades, of which 154 were resistant to neutralization by CD4-induced antibodies $17 \mathrm{~b}$ and $48 \mathrm{~d}$, the V3-directed antibodies 447-52D and 3074, and antibody F105, which recognized open conformations of Env (Supplementary Fig. $3 c$ and Supplementary Table $3 a$ ). Notably, vFP1.01 neutralized 18 strains at $50 \mu \mathrm{g} / \mathrm{mL}(8.2 \%$ breadth on 208-strain panel; $7.7 \%$ breadth on 154-resistant strain panel), while vFP7.04 neutralized 20 strains at $50 \mu \mathrm{g} / \mathrm{mL}$ (9.6\% breadth on 208-strain panel; $8.3 \%$ breadth on 154-resistant strain panel). vFP5.01, by contrast, neutralized only two strains, both sensitive to V3 and CD4-induced antibodies. Overall, neutralization by these first-generation monoclonal antibodies was of limited breadth and potency, although the best vFP antibodies could neutralize selected diverse strains of HIV-1.

Disparate antibody-bound FP conformations. To provide insight into the structural basis for neutralization by the first-generation vaccine-elicited antibodies, we determined crystal structures for the antigen-binding fragment (Fab) of vFP1.01 and vFP5.01 antibodies in complex with the eight $\mathrm{N}$-terminal residues of FP (Ala512-Phe519) at 2.0- and 1.5- $\AA$ resolutions, respectively (Supplementary Table 4a and Supplementary Fig. 4; also see expanded views in Fig. 2a,b). 


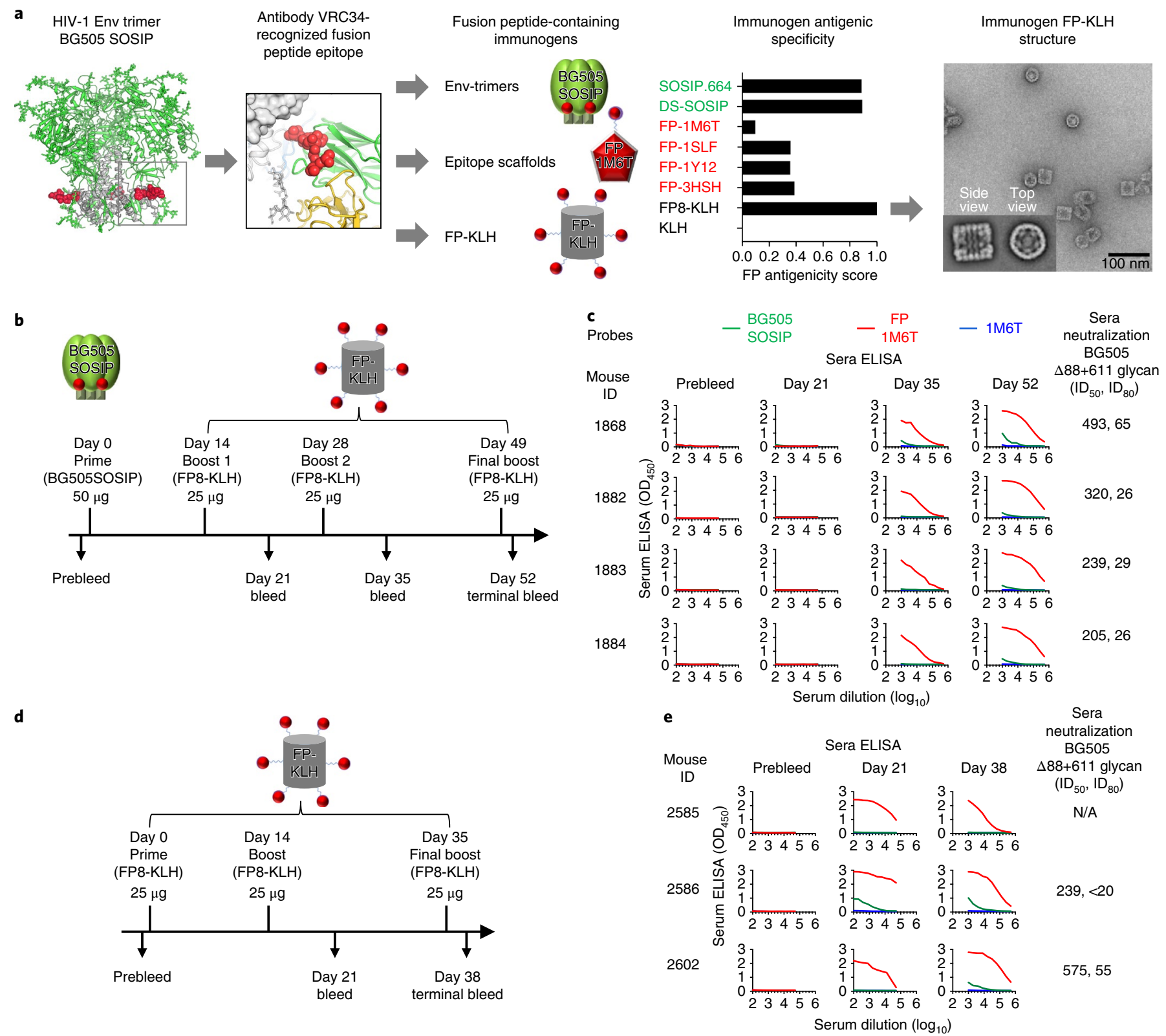

Fig. 1 | Design, properties, and immunogenicity of FP immunogens based on the epitope of antibody VRC34.01. a, Structure-based design, antigenic characteristics, and EM structure of FP immunogens. The glycosylated structure of the HIV-1 Env trimer is shown at far left, with exposed N terminus of FP highlighted in red. Subsequent images show site recognized by VRC34.01 antibody, schematics and antigenicity of FP immunogens, and negative stain EM of FP-KLH (see Supplementary Fig. 1 for details of FP antigenicity). For the EM study, $n=3$ independent experiments were performed with similar results. $\mathbf{b}$, Immunization regimen 1 . At day 52, mouse spleens were harvested and hybridomas created. $\mathbf{c}$, ELISA and neutralization of serum from regimen 1-immunized mice. Protein probes used for ELISA are defined in top row and include BG505 SOSIP.664 (green), FP-epitope scaffold based on PDB 1M6T (red), and 1M6T scaffold with no FP (blue). Column 1 defines mouse identification number and subsequent columns show ELISA and neutralization. ELISAs are shown as a function of serum dilution for prebleed, days 21, 35, and 52 (ELISA curves colored according to probe, with sera mostly unreactive with IM6T scaffold with no FP; $\mathrm{OD}_{450}$ is tthe optical density at the $450-\mathrm{nm}$ wavelength). Neutralization $\left(\mathrm{ID}_{50}\right.$, ID $\left.\mathrm{D}_{80}\right)$ values provided for day-52 serum; see Supplementary Fig. 2 a for neutralization details. d, Immunization regimen 2. At day 38, mouse spleens were harvested and hybridomas created. e, ELISA and serum neutralization of serum from regimen 2 -immunized mice, displayed as in $\mathbf{c}$.

The vFP1.01 co-crystals with FP were orthorhombic with four molecules per asymmetric unit, and in all four independent copies, Fab and FP assumed similar conformations, with FP adopting a curved structure with no intrachain-backbone hydrogen bonds. The $\mathrm{N}$ terminus of FP (Ala512) was buried between heavy and light chains, with the amino terminus forming a buried salt bridge with Glu34 $4_{\mathrm{vFP} 1.01-\mathrm{LC}}$, which was germline-encoded and shielded from solvent by a tetratyrosine cage, comprising tyrosines at residues $27 \mathrm{D}_{\mathrm{vFP} 1.01-\mathrm{LC}}, 32_{\mathrm{vFP} 1.01-\mathrm{LC}}$,
$96_{\mathrm{vFP1} 101-\mathrm{LC}}$, and $98_{\mathrm{vFP1} .01-\mathrm{HC}}$ (for clarity, we reference the molecule as a subscript for all molecules other than HIV Env by antibody name and HC or LC for heavy or light chain, respectively). The FP-main chain paralleled the curvature of the vFP1.01 complementaritydetermining region (CDR) H3, albeit with opposite orientation, up to residue Ile515, which packed against the body of the heavy chain before extending from antibody into the main body of the trimer with Gly516-Phe519 (Supplementary Fig. 4a,b). 
a

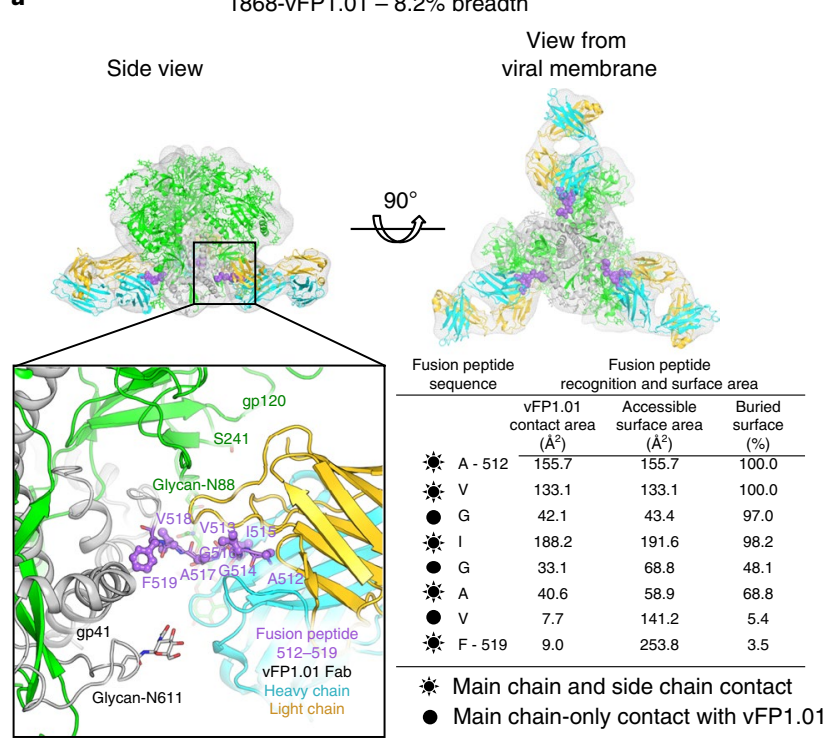

b

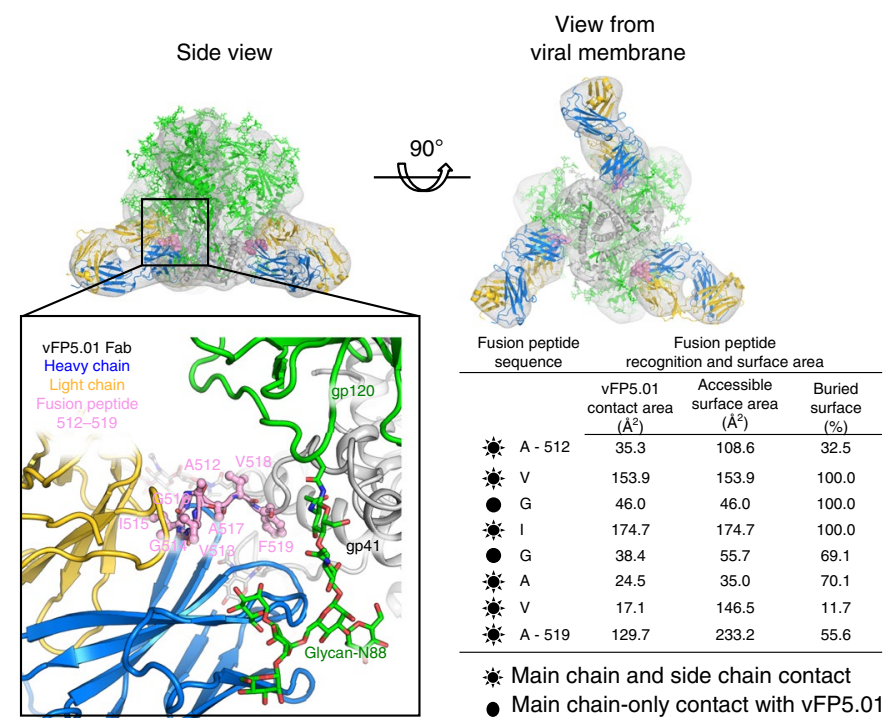

d

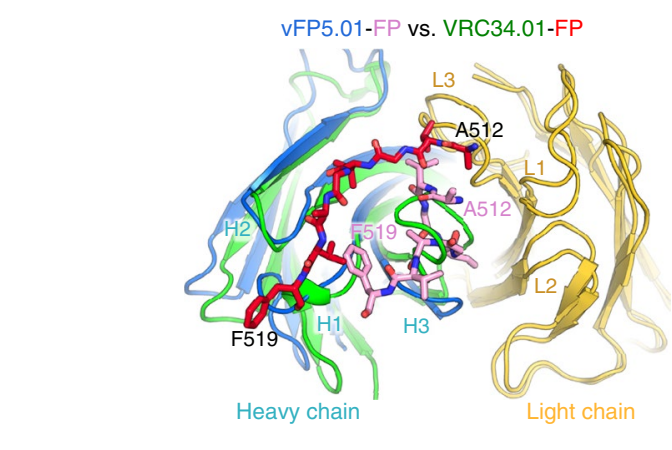

e

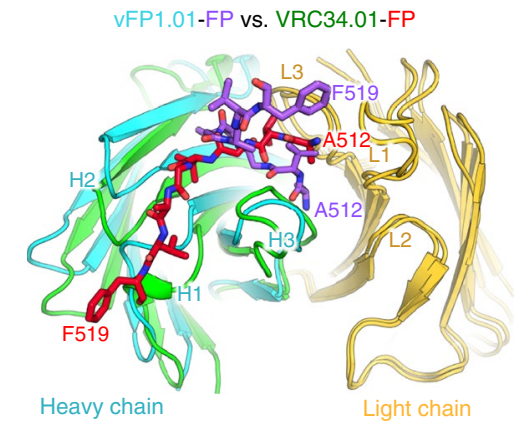

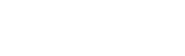
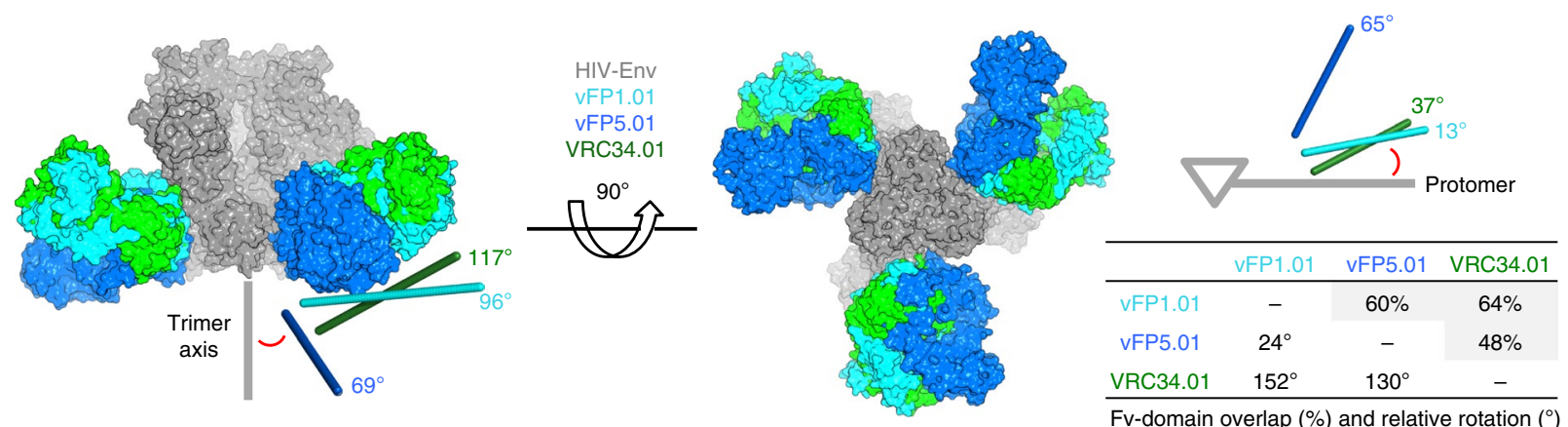

Fv-domain overlap (\%) and relative rotation $\left({ }^{\circ}\right)$

Fig. 2 | FP assumes disparate antibody-bound conformations, with neutralization restricted to a select angle of trimer approach. a, Structural definition of vFP1.01 recognition. Top: cryo-EM reconstruction at 8.6- $\AA$ resolution (density shown in gray) of Fab vFP1.01 in complex with BG505 DS-SOSIP trimer. Expanded view: crystal structure of Env trimer and FP-bound Fab vFP1.01 at 2.0- $\AA$ resolution, as modeled into the cryo-EM map by rigid-body docking. Env trimer for gp120 (green) and gp41 (gray), Fab vFP1.01 heavy chain (cyan) and light chain (yellow), and FP N terminus (purple). Surface areas provided for $\mathrm{N}$-terminal region of FP. $\mathbf{b}$, As in $\mathbf{a}$, but for vFP5.01 (blue and yellow) and FP (pink). Note that the angle of the lower right Fab differs from the angles of the other two. c, Comparison of FP bound by vFP1.01 vs. VRC34.01, with antibody shown in ribbons and FP in stick representation. d, As in c, but for vFP5.01. e, Angle of recognition and Fv-domain overlap for vFP1.01, vFP5.01, and VRC34.01. Measured angles (red) are between antibody angle of approach and Env-trimer axis (left, with viral membrane located below trimer) and between antibody angle of approach parallel to viral membrane and Env protomer (right, looking down trimer axis toward viral membrane).

The vFP5.01 co-crystals with FP were monoclinic, with one molecule per asymmetric unit. vFP5.01 bound FP at the interface of heavy and light chains, with the peptide adopting an overall hook structure: starting with a surface-exposed Ala512, dipping into the hydrophobic antibody interface with aliphatic side chains of Val513 and Ile515 anchoring the FP N terminus, before turning at Gly516 and extending from antibody toward Env (Supplementary Fig. 4c,d).
Comparison of antibody-bound crystal structures indicated substantial differences in FP conformation (Fig. 2c,d). While antibodies VRC34.01, vFP1.01, and vFP5.01 recognized the $\mathrm{N}$ terminus of FP by using a similar region at the CDR-H3-CDR-L3 interface of each antibody, the conformations of the antibody-bound FPs were substantially different: the vFP1.01-bound FP formed a U-shaped structure focused at the heavy-light interface, vFP5.01 bound FP in 

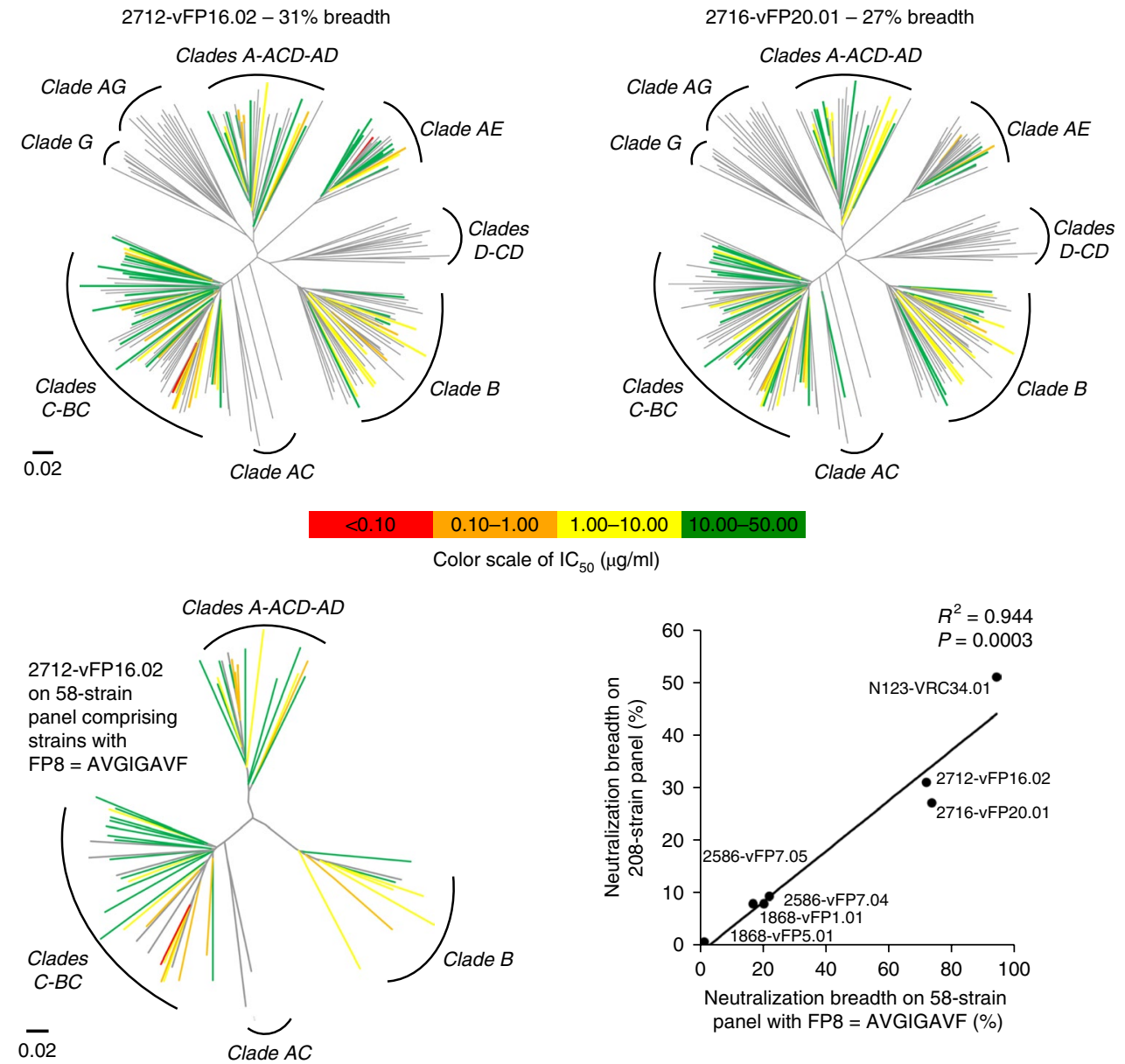

Fig. 3 | FP-directed vaccine-elicited antibodies neutralize up to $31 \%$ of HIV-1. Neutralization dendrograms display the diversity of tested viral strains, with branches colored according to neutralization potency (non-neutralized branches shown in gray). Top row: 208-strain panel for vaccine-elicited antibodies vFP16.02 and vFP20.01. Bottom left: 58-strain panel, displaying only branches with FP8 = AVGIGAVF. Bottom right: comparison of breadth on 58- and 208-strain panels shown with Pearson correlation ( $n=7$ antibodies).

an extended conformation to interact with CDR H3, and VRC34.01 bound FP in an extended conformation to interact with CDR H1. To place these disparate antibody-recognized conformations of FP into a more general context, we used principal component analysis to cluster $\mathrm{N}$-terminal FP conformations from a molecular dynamics simulation of fully glycosylated HIV-1 Env. Four prevalent clusters of FP conformations were observed (Supplementary Fig. 4e,f), with FP-directed antibodies recognizing disparate but prevalent conformations of FP.

Restricted angle of approach for FP-directed neutralization. To position the vFP1.01 and vFP5.01 structures with FP into the context of the HIV-1 Env trimer, we collected cryo-EM data for these FP-directed antibodies complexed to the BG505 SOSIP trimer. With Fab vFP1.01, approximately 14,000 particles yielded an 8.6- $\AA$ resolution reconstruction after threefold averaging (Supplementary Fig. 5a-e); the resulting structure (Fig. 2a) showed three Fabs laterally interacting with the Env trimer. With Fab vFP5.01, several particle classes were observed yielding reconstructions at 14.7- and 19.6- $\AA$ resolutions (Supplementary Fig. 5f-h); these asymmetric reconstructions indicated each of the vFP5.01 Fabs to approach Env differently (Fig. 2b).

To provide insight into recognition of the Env trimer, we analyzed the approach angle of the FP-recognizing antibodies (Fig. 2e). Overall, the approach of antibodies directed primarily to FP and capable of neutralizing diverse HIV-1 strains was similar, suggesting restrictions on trimer approach for effective FP-directed neutralization.

Considerations for improved immunizations. Analysis of the first-generation antibodies indicated that effective FP-directed neutralization would occur preferentially at a restricted angle of trimer approach, thereby suggesting that boosting with Env trimer might elicit improved neutralization. We sought additional clues from analysis of the first-generation vFP antibodies to improve FP immunization.

To provide insight into sequence requirements for neutralization, we created a panel of peptides comprising Ala and Gly mutants of the $\mathrm{N}$ terminus of $\mathrm{FP}$ and screened for recognition by vaccineelicited antibodies and by VRC34.01 (Supplementary Fig. 6a and Supplementary Table 5). The Ala-Gly mutants only affected vFP1.01 recognition if they occurred within the first four residues of FP (512-515). For vFP5.01, a more extensive range was observed, with alterations to Ala-Gly at residues 513, 514, 515, 516, and 519 affecting recognition. VRC34.01 recognition was intermediate between vFP1.01 and vFP5.01, being sensitive to changes at 512, 513,515 , and 516 , and partially sensitive to changes at 518 and 519 . These results indicated a preference for $\mathrm{N}$-terminal residues for effective neutralization, thereby suggesting that N-terminal focusing might improve neutralization of the vaccine-elicited antibodies. 


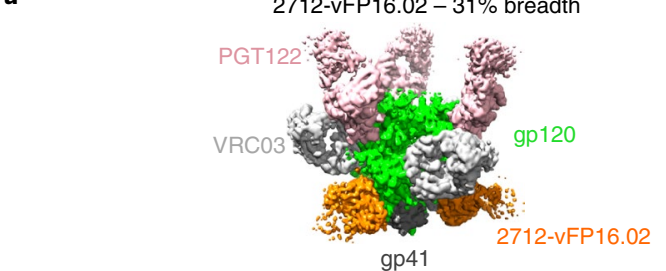

b

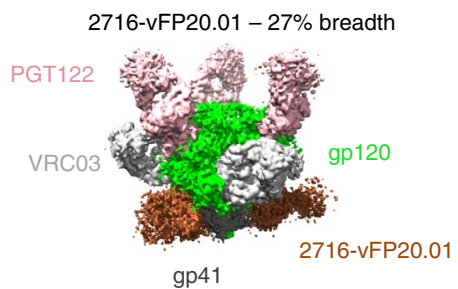

C

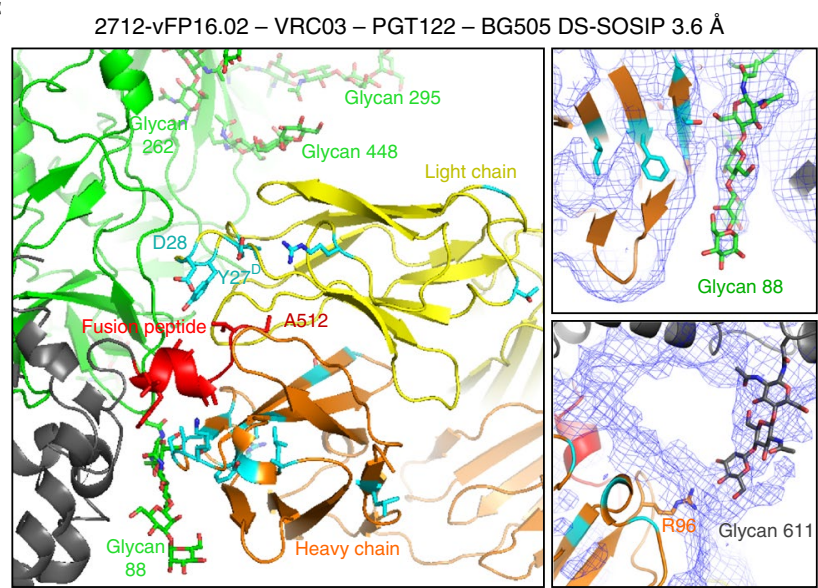

d
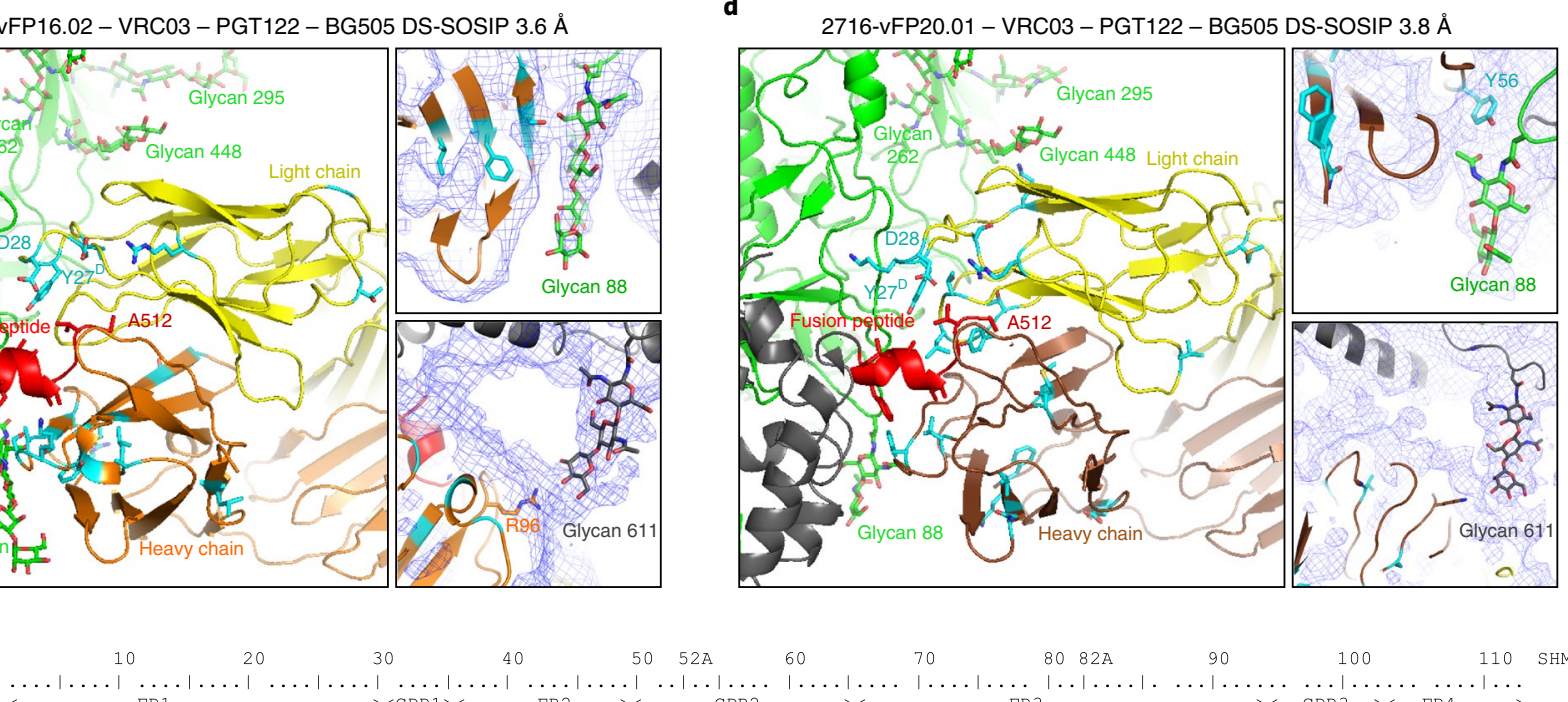

$30 \quad 40$

40

$50 \quad 52 \mathrm{~A}$

60

70

$80 \quad 82 \mathrm{~A}$

90

100

110 SHM (\%)

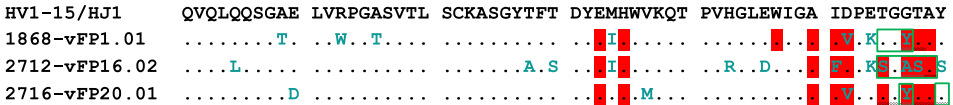

2716-vFP20.01

Light chain

KV1-117/KJ2

1868-vFP1. 01

2712-vFP16.02

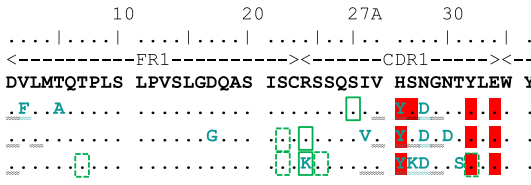

40

50

$50 \quad 60$

$\begin{array}{ll}60 & 70\end{array}$

$70 \quad 80$
$\ldots 1 \ldots$

100
$\ldots|\ldots \ldots \ldots+\ldots| \ldots$

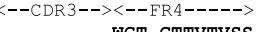

LR NYWYFDV $\ldots$. $\ldots \ldots$ P 5.5

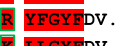

2716-vFP20.01

Fig. 4 | Substantial glycan contacts by vaccine-elicited FP-directed antibodies. a, Cryo-EM map of quaternary complex with antibody 2712-vFP16.02, segmented by components at a contour level that allowed visualization of Fv domains of antibodies (also see Supplementary Figs. 9 and 10). b, As in a, but with 2716-vFP20.01. c, Details of vFP16.02 interaction. Right: experimental EM density in blue mesh, with contour level adjusted to allow visualizing of partially ordered glycan. Residues altered by SHM highlighted in cyan. Antibody vFP16.02 contacts both FP and neighboring glycans to achieve $31 \%$ breadth. d, As in c, but for vFP20.01. e, Sequence alignment of vaccine-elicited FP-directed antibodies and origin genes. FP contacts (red highlight), glycan contacts (green rectangles), and SHM (cyan font) are highlighted. Additional Env contacts are indicated by double underlining. Because the density from the cryo-EM reconstructions was not always sufficient to allow for atomic-level fitting, contacts shown with dotted green rectangles were inferred.

Although we did not observe a substantial improvement in neutralization titers upon Env priming (Fig. 1), we nevertheless analyzed the degree of affinity maturation for vFP1-class antibodies, as this would lend insight into the induction of these antibodies. We identified vFP1-class antibodies from three additional mice, two of them (1882 and 1883) primed with Env trimer and one (2602) immunized with only FP-KLH (Supplementary Fig. 7a). Notably, vFP1-class antibodies primed with Env trimer showed higher SHM with statistical significance $(P=0.0046$; Supplementary Fig. $7 b)$. Thus, while Env-trimer priming did not improve neutralization, it did appear to prime vFP1-class antibodies.

Second-generation FP-directed antibodies neutralize up to $31 \%$ of HIV-1 strains. To elicit improved FP-directed antibodies, we tested 11 immunization regimens on $16 \mathrm{C} 57 \mathrm{BL} / 6$ mice (Supplementary Fig. 8a). The vaccine regimens included a BG505 SOSIP trimer prime and various FP-KLH boosts, along with a BG505 DS-SOSIP boost on a subset of 5 of the mice. The FP-KLH boosts used different lengths of FP, ranging from FP6 through FP10, which incorporated 6-10 residues from the N-terminal FP sequence of strain BG505. Serum neutralization titers for the 5 trimer-boosted animals were especially improved, reaching an $\mathrm{ID}_{50}$ as high as 77,379 (mouse 2716$)$ for the glycan-deleted $(\Delta 88+611)$ BG505 (Supplementary Fig. 8a,b). We tested the serum neutralization for the 5 trimer-boosted mice on a panel of 10 selected wildtype strains, which were chosen for their sensitivity to VRC34.01 or vFP1.01 and to encompass divergent HIV-1 clades (Supplementary Figs. $2 \mathrm{~b}$ and $8 \mathrm{c}$ ). While modest neutralization from these 5 sera was observed against the selected strains, serum from mouse 2716 achieved $\mathrm{ID}_{50}$ neutralization against all ten of these strains and $\mathrm{ID}_{80}$ against seven of them. Analysis by peptide competition indicated neutralization by the mouse 2716 serum to be targeted primarily to FP (Supplementary Fig. 2c).

For each of the 16 immunized C57BL/6 mice, we isolated and characterized monoclonal antibodies. These could be parsed into 21 lineages, vFP12-vFP32 (Supplementary Table 1a-c), and we 
a Guinea pig FP immunization

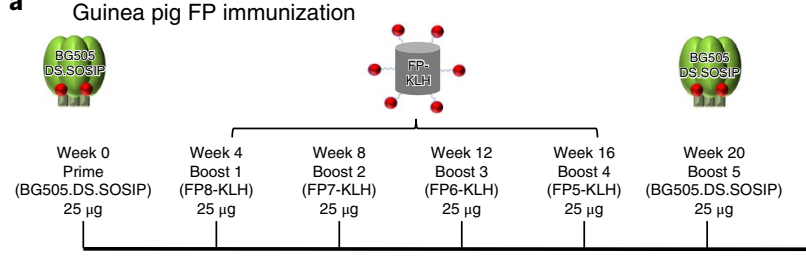

Neutralization of guinea pig FP immunization study week 28 plasma

\begin{tabular}{llllllllllllllll}
\hline Clade & A & A & B & B & C & AE & BC & C & C & A & A & A & A
\end{tabular}

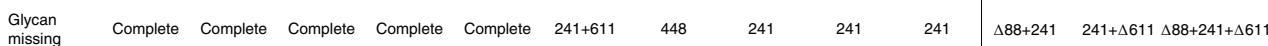
\begin{tabular}{lllllllllllll} 
Viruses & KER2008.12 & Q23.17 & 3988.25 & BL01.DG & 286.36 & CNE56 & CNE19 & 25710-2.430077.V1.C16 & BG505 & BG505 $\Delta 88$ BG505 $\Delta 611$ & BG505 \\
\hline $88+611$
\end{tabular}

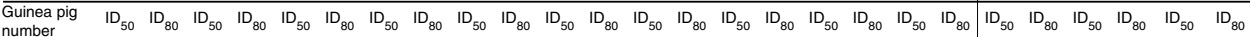
\begin{tabular}{lllllllllllllllllllllllll|l|lll} 
CGP701-1 & $<20$ & $<20$ & $<20$ & $<20$ & $<20$ & $<20$ & $<20$ & $<20$ & $<20$ & $<20$ & 70 & $<20$ & $<20$ & $<20$ & 45 & $<20$ & $<20$ & $<20$ & 81 & $<20$ & 145 & 22 & 6,149 & 953 & 29,545 & 8,365
\end{tabular}



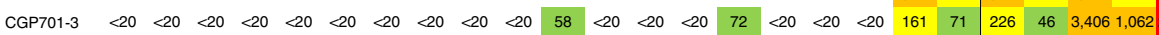

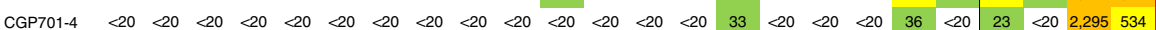

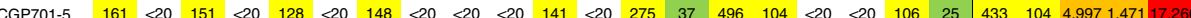
$<20 \begin{array}{ccc}20-100 & 100- & 1000- \\ 1,000 & 10,000\end{array}$

c NHP FP immunization

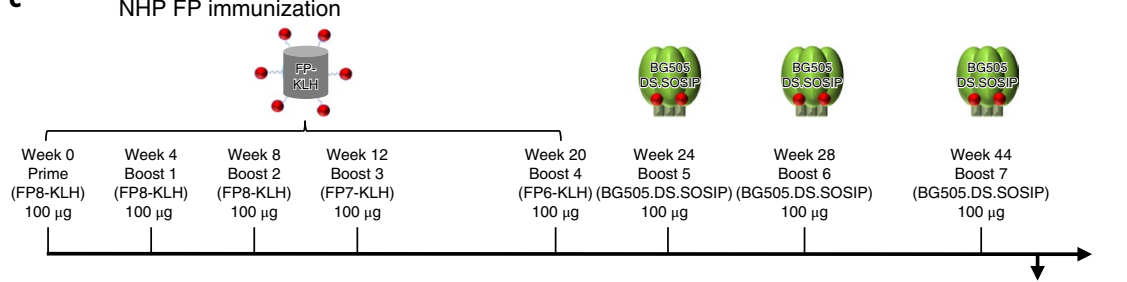

Key:
FP8 $=$ AVGIGAVF FP7 $=$ AVGIGAV
FP6 $=$ AVGIGA

Neutralization of NHP FP immunization study week 46 plasma

\begin{tabular}{|c|c|c|c|c|c|c|c|c|c|c|c|c|c|c|c|c|c|c|c|c|c|c|c|c|}
\hline & & & & & & & & & & & & & & & & & & & & & lasma & & & \\
\hline ade & A & & A & & $B$ & & $B$ & & $\mathrm{c}$ & c & A & $\mathrm{NE}$ & $B$ & $3 \mathrm{C}$ & & & $\mathrm{c}$ & $\mathrm{c}$ & A & A & & A & & A \\
\hline ssir & $\mathrm{mp}$ & ple & Com & plete & omp & plete & Comp & & Comp & iple & 41. & +6 & 4 & 48 & & 241 & 24 & 41 & 241 & $88+$ & +2 & $41+\Delta 611 \Delta$ & $\Delta 88+24$ & $-\Delta 611$ \\
\hline uses & KER2O & 008.12 & Q23 & & 3988. & 8.25 & BL01 & 1.DG & 286. & 5.36 & $\mathrm{CN}$ & E56 & $\mathrm{CN}$ & E19 & 25711 & $0-2.43$ & 0077.V & V1.C16 & BG505 & BG505 & $5 \Delta$ & 11 & & \\
\hline & $\mathrm{ID}_{50}$ & $\mathrm{ID}_{80}$ & $\mathrm{ID}_{50}$ & $\mathrm{ID}_{80}$ & $1 \mathrm{I}_{50}$ & $1 \mathrm{I}_{80}$ & $\mathrm{ID}_{50}$ & $\mathrm{ID}_{80}$ & $\mathrm{ID}_{50}$ & $\mathrm{ID}_{80}$ & $\mathrm{ID}_{50}$ & $1 \mathrm{D}_{80}$ & $\mathrm{ID}_{50}$ & $\mathrm{ID}_{80}$ & $\mathrm{ID}_{50}$ & $\mathrm{ID}_{80}$ & $\mathrm{ID}_{50}$ & $\mathrm{ID}_{80}$ & $\mathrm{ID}_{50} \quad \mathrm{ID}_{80}$ & \begin{tabular}{|l|l|}
0 & $1 D_{50}$
\end{tabular} & $\mathrm{ID}_{80}$ & $\mathrm{ID}_{50} \quad \mathrm{ID}_{80}$ & $\mathrm{ID}_{50}$ & $\mathrm{ID}_{80}$ \\
\hline & $<20$ & 20 & $<20$ & $<20$ & $<20$ & $<20$ & $<20$ & $<20$ & $<20$ & $<20$ & $<20$ & $<20$ & $<20$ & $<20$ & $<20$ & $<20$ & $<20$ & $<20$ & $113<20$ & 167 & $<20$ & $\begin{array}{ll}172 & 25\end{array}$ & 604 & 70 \\
\hline EPE & $<20$ & $<20$ & 31 & $<20$ & $<20$ & $<20$ & $<20$ & $<20$ & $<20$ & $<20$ & $<20$ & $<20$ & 85 & 20 & 104 & 28 & $<20$ & $<20$ & $\begin{array}{ll}180 & 23\end{array}$ & 231 & 28 & $806 \quad 196$ & 2,365 & 500 \\
\hline 110 & 24 & $<20$ & 145 & 20 & $<20$ & $<20$ & 91 & 24 & 103 & 23 & $<20$ & $<20$ & 33 & $<20$ & 741 & 170 & 98 & $<20$ & $205<20$ & 450 & 60 & $6,1311,571$ & 2501 & 3,998 \\
\hline 40 & $<20$ & $<20$ & $<20$ & $<20$ & $<20$ & $<20$ & $<20$ & $<20$ & $<20$ & $<20$ & $<20$ & $<20$ & 26 & $<20$ & 63 & $<20$ & $<20$ & $<20$ & $22<20$ & 38 & $<20$ & $\begin{array}{ll}913 & 187\end{array}$ & 4,772 & 658 \\
\hline $\mathrm{PV}$ & $<20$ & $<20$ & 29 & $<20$ & $<20$ & $<20$ & $<20$ & $<20$ & $<20$ & $<20$ & $<20$ & $<20$ & 28 & $<20$ & 201 & 49 & $<20$ & $<20$ & $66<20$ & 77 & $<20$ & $\begin{array}{l}1,231 \quad 292\end{array}$ & 5,211 & 1,386 \\
\hline
\end{tabular}

$<2020-100 \begin{array}{ccc}100- & 1,000 \\ 1,000 & 10,000\end{array}$ b

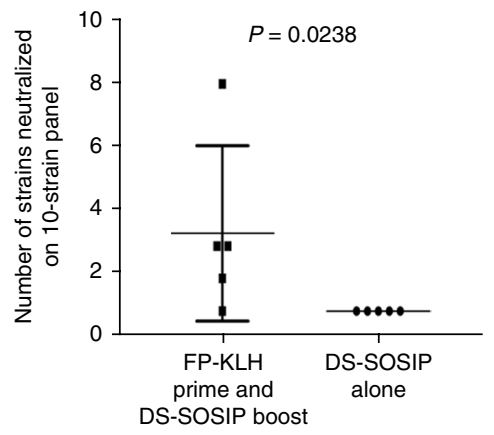

d



Fig. 5 | Immunization of guinea pigs and NHPs with FP-coupled carriers and DS-SOSIP trimer elicits heterologous neutralizing responses. a, Elicitation of serum neutralizing responses in guinea pigs. Immunization regimen and week 28 serum ID ${ }_{50}$ titers as measured on a panel of 10 wild-type strains, 5 with complete glycans around FP and 5 naturally missing glycans at sites shown. Also shown are titers for $\Delta 88, \Delta 611$, and $\Delta 88+611$ glycan-deleted variants of BG505. b, Plot comparing guinea pig-serum neutralization breadth for FP-KLH prime + DS-SOSIP trimer-boost regimen versus DS-SOSIP-alone regimen (one-tailed Mann-Whitney test; see Supplementary Fig. 7 for DS-SOSIP alone immunizations at 0, 4, and 16 weeks; $n=5$ animals for each group).

c, Elicitation of heterologous neutralizing responses in rhesus macaques. Immunization scheme and week 46 serum titers assessed and displayed as in a. d, Plot comparing NHP serum neutralization breadth for immunization regimens, displayed as in $\mathbf{b} ; n=5$ animals for each group.

screened each of the vFP antibodies against two wild-type viruses, clade A BG505 and clade B 3988.25; 24 antibodies showed wild-type neutralization, with most neutralizing $70 \%$ or more of the ten-strain panel (Supplementary Table 3b).

We chose two antibodies, 2712-vFP16.02 and 2716-vFP20.01, for in-depth assessment (vaccine-elicited FP antibodies were named for mouse ID-lineage.clone, with antibody 2716-vFP20.01 being clone 01 from lineage vFP20 isolated from mouse ID 2716). Notably, on the 208-strain panel, these two antibodies achieved $31 \%$ and $27 \%$ neutralization breadth, respectively, when assessed at a maximum $\mathrm{IC}_{50}$ level of $50 \mu \mathrm{g} / \mathrm{mL}$ (Fig. 3 and Supplementary Table 3a). The breadth and potency of these two vFP antibodies were similar to that of antibody 2G12 (Supplementary Fig. 11b), which has been shown to delay rebound and to induce selection pressure on the viral quasispecies when passively infused ${ }^{42-44}$. Neutralization extended to multiple clades, including clades A, B, and C, but for several clades, includ- ing clades $\mathrm{AC}, \mathrm{AG}, \mathrm{D}, \mathrm{CD}$, and $\mathrm{G}$, no neutralization was observed. In the neutralized clades, the immunized FP8 sequence was generally present, whereas in the non-neutralized clades, this FP8 sequence was not present (in clade AE, the prevalent FP8 sequences included changes in the C-terminal region of FP8 (Supplementary Table 6), which were apparently tolerated by both vFP16.02 and vFP20.01 antibodies). We observed strong correlation between FP-directed antibody breadth on the 208-strain panel and the 58-strain panel with identical FP8 sequences, indicating overall breadth of the vFP antibodies to correlate with the ability to neutralize strains with the most prevalent FP8 sequence (Fig. 3). These results provide proof of principle for the ability of FP-epitope focusing to induce FP-directed antibodies with promising neutralization breadth.

Structures and Env interactions of second-generation antibodies vFP16.02 and vFP20.01. To gain insight into the cross-clade 


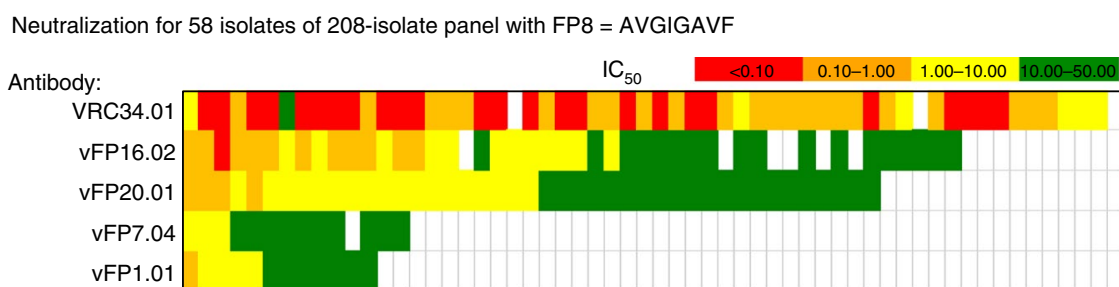

Plasma:

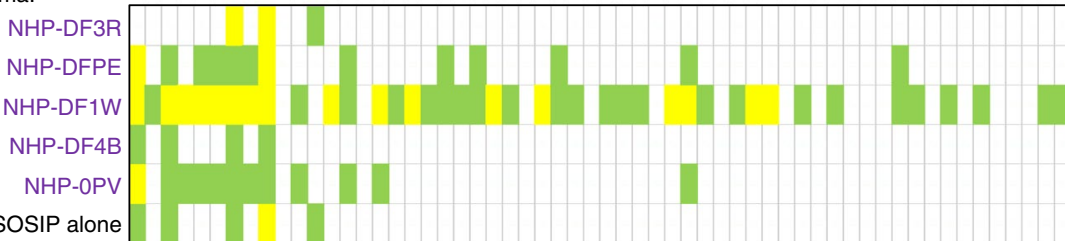

\begin{tabular}{cccc}
$\begin{array}{c}\text { Number } \\
\text { neutralized } \\
\text { on 58-strain } \\
\text { panel IC }\end{array}$ & $\begin{array}{c}\text { Breadth on } \\
\text { 208-strain } \\
\text { panel }\end{array}$ & $\begin{array}{c}\text { Breadth on } \\
\text { 154-resistant } \\
\text { isolate panel }\end{array}$ & $\begin{array}{c}\text { Number } \\
\text { neutralized } \\
\text { on 58-strain } \\
\text { panel IC }\end{array}$ \\
\hline 55 & $51 \%$ & $50 \%$ & 42 \\
42 & $31 \%$ & $29 \%$ & 20 \\
43 & $27 \%$ & $25 \%$ & 13 \\
13 & $9.6 \%$ & $8.3 \%$ & 3 \\
12 & $8.2 \%$ & $7.7 \%$ & 2 \\
3 & - & - & 1 \\
12 & - & - & 3 \\
41 & - & - & 13 \\
4 & - & - & 0 \\
12 & - & - & 2 \\
5 & - & - & \\
\hline
\end{tabular}

$$
\text { 个BG505 }
$$

b
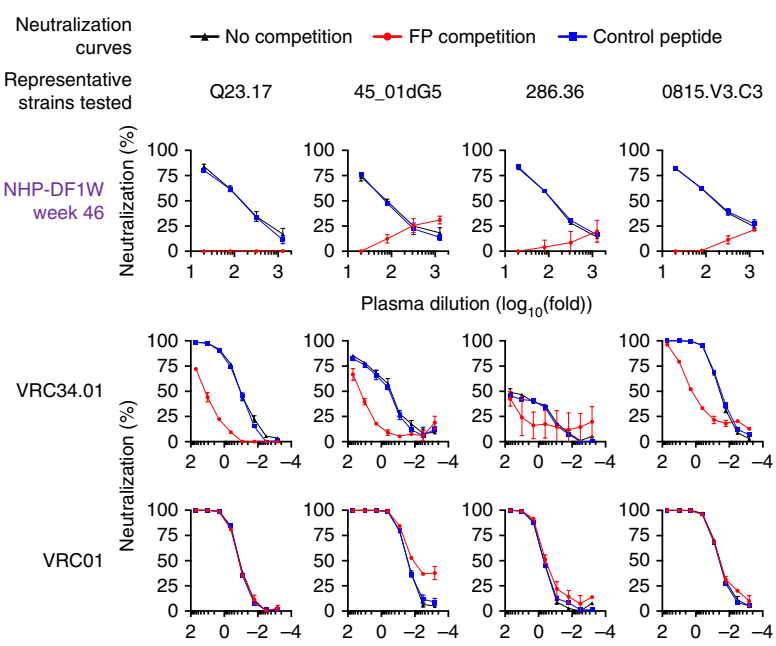

Antibody concentration $\left(\log _{10}(\mu \mathrm{g} / \mathrm{mL})\right)$ c NHP DF1W week 46 plasma neutralization on 58 -strain panel with FP8 = AVGIGAVF

\section{Clades $A-A C D-A D$}



d Neutralization fingerprint on 58-strain panel with FP8 = AVGIGAVF

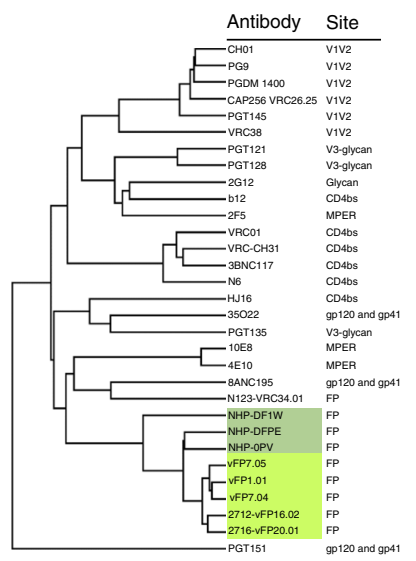

Fig. 6 | Patterns of neutralization indicate broad FP-directed responses in mice and NHP are related. a, Neutralization on a 58-strain panel, comprising strains from the 208-strain panel with the FP sequence AVGIGAVF matching both FP8-KLH and BG505 trimer immunogens. Top: IC 50 values for FP-directed antibodies. Bottom: ID $_{50}$ titers for NHP plasma: top 5 rows, FP-KLH primer + DS-SOSIP trimer-boost regimen at week 46, 2 weeks after third trimer boost; bottom row, DS-SOSIP alone regimen at week 18, 2 weeks after third trimer boost. Numbers of neutralized strains and neutralization breadths are shown. (IC 80 and ID 80 provided in Supplementary Table 3c and Supplementary Fig. 7.) b, Neutralization curves of NHP plasma (DF1W week 46) and control monoclonal antibodies (VRC34.01 and VRC01) on four representative strains in the presence of no peptide (black), FP (red), or an irrelevant Flag peptide (blue). Mean and s.d. of results from triplicated experiments shown ( $n=3$ independent experiments). c, Neutralization dendrogram $\left(I_{50}\right)$ for NHP DF1W week 46 plasma on 58-strain panel, showing location of representative strains tested in $\mathbf{b}$. d, Neutralization-fingerprint dendrogram calculated from 58-strain panel. Vaccine-elicited vFP antibodies (light-green background) and 3 NHP week-46 plasmas (dark-green background for 3 of 5 NHPs with sufficient neutralization to yield accurate fingerprint analysis) clustered next to each other (see also fingerprint dendrogram on 132 curated strains shown in Supplementary Fig. 11a).

breadth observed with vFP16.02 and vFP20.01 antibodies, we determined their crystal structures in complex with FP (Supplementary Fig. 4g,h) and their cryo-EM structures in complex with HIV-1 Env trimer (Fig. 4). The crystal structures with FP revealed highly similar recognition of residues 512-517, with vFP1-class antibodies constraining little of the FP conformation beyond residue 517 (Supplementary Fig. 4g,h and Supplementary Table 4a). The cryoEM data were collected on a quaternary complex with BG505 DS-SOSIP trimer bound by antibodies PGT122 and VRC03, in addition to vFP16.02 or vFP20.01; the added antibodies increased the size of the complex and provided fiducial markers allowing better particle visualization and alignment. The resultant reconstructions displayed resolutions of 3.6 and $3.8 \AA$, respectively, when calculated according to the FSC 0.143 gold-standard criterion with soft-edged masks from which flexible constant regions had been removed (Fig. 4, Supplementary Fig. 9a-f, and Supplementary Table 4b). In the reconstructions, we observed electron density for the vFP16.02 and vFP20.01 antibodies focused around the fusion peptide, with density becoming weaker farther from the fusion peptide site of recognition (Supplementary Fig. 10a,b).

Glycan contacts between vFP antibodies and Env trimer were observed (Fig. 4c,d), and SHM was observed to occur preferentially at the interface with Env, especially involving interactions with FP and with $N$-linked glycan (Fig. 4 e). In both antibody-Env complexes, glycans N448 and N611 displayed similar orientations, with glycan N448 buttressed by the light chain on one side and by glycan N295 on 
the other, and with glycan N611 projecting from a neighboring Env protomer directed toward the antibody heavy chain. Glycan N88 also displayed ordered density for its protein-proximal sugars, though this differed in the two antibody complexes: in vFP16.02, substantial ordering was observed, with the glycan lodged between gp41 and the heavy chain (Fig. 4c); in vFP20.01, glycan N88 was less ordered and assumed a substantially different conformation to accommodate the SHM-altered Gly56 Tyr $\mathrm{vFP20.01-HC}_{\mathrm{HC}}$ side chain (Fig. 4d). We note that even though removal of glycans neighboring FP (for example, glycan 611) improved neutralization by these antibodies, resistance analysis (Supplementary Table 7) indicated that neutralization in the 208-strain panel was not dependent on the absence of FP-neighboring glycans. Overall, the structures indicated murine vFP antibodies with promising breadth to substantially accommodate, if not partially recognize, FP-proximal $N$-linked glycan.

Translation of murine vaccine regimens into guinea pigs and nonhuman primates. We extracted insights from the murine immunization regimens, such as trimer boost and FP N-terminal focusing, and applied these to guinea pigs and rhesus macaques (nonhuman primates; NHPs). We immunized 5 guinea pigs using a scheme modeled on the mouse-2716 immunization regimen (Fig. 5a). At week 28, 4 of 5 guinea pigs showed some heterologous virus neutralization on the ten-strain panel. When compared to guinea pigs immunized with DS-SOSIP alone (Supplementary Fig. 7e), the ten-strain breadth induced by the FP-KLH-primed DS-SOSIP boost was significantly higher than that induced by DS-SOSIP alone (Fig. 5a,b).

For NHP, we also used a regimen similar to that used for mouse 2716, but omitted the initial trimer prime and used three trimer boosts so that we could compare to NHPs immunized with trimer only. We used a total of 5 FP-KLH primes with FP peptides of lengths eight, eight, eight, seven, and six. At week 46, 4 of 5 NHP plasmas showed heterologous virus neutralization on the ten-strain panel (Fig. $5 \mathrm{c}, \mathrm{d}$ ). As seen in the GP immunization study, this breadth of neutralization by NHP plasma was higher than that induced by immunization with DS-SOSIP alone (Supplementary Fig. 7f) ${ }^{45}$.

We further assessed week-46 plasma from all 5 NHP on a 58-strain subset of the 208-strain panel, restricted to strains for which the sequence of FP was AVGIGAVF, the most prevalent FP sequence and the sequence in the FP8-KLH and BG505 DS-SOSIP immunogens. At a 1:20 dilution, 3 of 5 NHP plasmas showed substantial neutralization breadth, with the broadest, NHP DF1W, achieving $\mathrm{ID}_{50}$ neutralization on 41 of 58 strains $(70 \%)$ and $\mathrm{ID}_{80}$ neutralization of 13 of 58 strains (22\%; Fig. 6a and Supplementary Table 3c). Neutralization competition of the NHP DF1W plasma tested against neutralization-resistant HIV-1 strains with complete glycan around FP from four different clades indicated that a linear FP, matching the immunogen sequence, could adsorb virtually all of the neutralizing activity (Fig. 6 b and Supplementary Fig. 2d). A dendrogram of these 58 Env strains (Fig. 6c) indicated that neutralization was distributed over several clades, consistent with the distribution of the immunized FP8 sequence in HIV-1. Additionally, the neutralization fingerprints of the three NHP plasmas, with substantial breadth clustered next to the neutralization fingerprints of the murine vFP1-class antibodies (Fig. 6d), provided strong indication for the similarity of induced murine and NHP immune responses.

\section{Discussion}

The vaccine elicitation of antibodies capable of neutralizing diverse strains of HIV-1 has been a goal of HIV-1 research for over 30 years. While substantial strides have been made in the creation of prefusion-stabilized Env trimers ${ }^{40,41,46-49}$, responses elicited by these trimers have been primarily strain-specific. Here we show that focusing the immune response on the exposed $\mathrm{N}$-terminal residues of FP elicits antibodies of promising neutralization breadth in multiple vaccine-test species. Several factors contributed to the successful elicitation of neutralizing antibodies. First, antibody VRC34.01 ${ }^{31}$ defined a precise target-not the entire FP, but only exposed $\mathrm{N}$-terminal residues - upon which to focus. Second, glycan-deleted viruse ${ }^{50}$ provided a readout sensitive enough to detect initial weak serum responses. Third, we found prefusion-closed Env trimers ${ }^{40}$ to be necessary for boosting serum responses. Most important, however, may be the characteristics of the target site-the FP N terminus-a conserved and exposed site of vulnerability, for which high conformational variability was compatible with broad neutralization (Supplementary Figs. 4e and 11c-g).

FP-directed antibodies of substantial breadth have been reported for other viral pathogens, including influenza A virus ${ }^{51,52}$, Ebola virus $^{53-55}$, and Lassa virus ${ }^{56}$. Although the fusion peptides of these pathogens are substantially different from that of HIV-1, the generality of FP-targeted neutralizing antibodies suggests that characteristics of FP may be especially suited to vaccine targeting. Such characteristics include its conservation (related to function), its chemistry (hydrophobic residues afford high binding energy), and its exposure (FP is often proximal to a site of proteolytic activation).

Is there a neutralization limit or constraint for $\mathrm{vFP}$ antibodies based on viral properties (for example, related to strain-specific conformation or accessibility) other than the diversity of FP itself? A strong correlation was observed between Env trimer affinity and neutralization breadth (Supplementary Figs. 6b,c and 11d), suggesting that enhanced Env-trimer affinity would lead to increased breadth. Indeed, at tenfold higher concentration $(500 \mu \mathrm{g} / \mathrm{mL})$, vFP16.02 neutralized 97\% (56 of 58 strains) with the immunized FP8 sequence, and $47 \%$ of the 208-strain panel (97 of 208 strains; Supplementary Table $3 a)$. Thus, for strains with the same sequence as used in the FP immunizations, there did not appear to be an intrinsic limit to elicited FP-directed breadth.

Finally, is the observed elicitation of vFP-neutralizing antibodies in animal models translatable to humans? While broadly neutralizing antibodies against HIV-1 have been induced in animals such as cows $^{57}$ and llamas ${ }^{58}$, these antibodies rely on species-specific characteristics $^{59,60}$ (knob domains for cow and unpaired heavy chains for llama), which are not present in human antibodies. By contrast, there does not appear to be an intrinsic barrier to eliciting vFP antibodies in humans: genetic analysis indicates that humans have $\mathrm{V}$-genes with some similarity to the germline genes of the murine vFP1-antibody class (Supplementary Fig. 12a,b), FP-directed antibodies can often be detected in HIV-1-infected donors by ELISA ${ }^{31}$, and FP-directed antibodies showed almost no indication of polyreactivity (Supplementary Fig. 12c,d). Overall, our results provide proof of principle for the ability of epitope-based vaccine design to induce FP-directed antibodies with neutralization breadth and indicate that the exposed $\mathrm{N}$ terminus of FP is a site of exceptional HIV-1 vaccine promise.

\section{Methods}

Methods, including statements of data availability and any associated accession codes and references, are available at https://doi. org/10.1038/s41591-018-0042-6.

Received: 24 January 2018; Accepted: 19 March 2018; Published online: 4 June 2018

\section{References}

1. Korber, B. et al. Timing the ancestor of the HIV-1 pandemic strains. Science 288, 1789-1796 (2000).

2. UNAIDS. Global AIDS Update 2016, UNAIDS http://www.unaids.org/en/ resources/documents/2016/Global-AIDS-update-2016 (2016).

3. Hraber, P. et al. Prevalence of broadly neutralizing antibody responses during chronic HIV-1 infection. AIDS 28, 163-169 (2014).

4. Mascola, J. R. \& Haynes, B. F. HIV-1 neutralizing antibodies: understanding nature's pathways. Immunol. Rev. 254, 225-244 (2013). 
5. Seaman, M. S. et al. Tiered categorization of a diverse panel of HIV-1 Env pseudoviruses for assessment of neutralizing antibodies. J. Virol. 84, 1439-1452 (2010).

6. Kwong, P. D. \& Mascola, J. R. Human antibodies that neutralize HIV-1: identification, structures, and B cell ontogenies. Immunity 37, 412-425 (2012).

7. Burton, D. R. et al. A blueprint for HIV vaccine discovery. Cell Host Microbe 12, 396-407 (2012).

8. Chen, L. et al. Structural basis of immune evasion at the site of CD4 attachment on HIV-1gp120. Science 326, 1123-1127 (2009).

9. Zhou, T. et al. Structural Repertoire of HIV-1-neutralizing antibodies targeting the CD4 supersite in 14 donors. Cell 161, 1280-1292 (2015)

10. Scheid, J. F. et al. Sequence and structural convergence of broad and potent HIV antibodies that mimic CD4 binding. Science 333, 1633-1637 (2011).

11. $\mathrm{Wu}, \mathrm{X}$. et al. Rational design of envelope identifies broadly neutralizing human monoclonal antibodies to HIV-1. Science 329, 856-861 (2010).

12. Wu, X. et al. Focused evolution of HIV-1 neutralizing antibodies revealed by structures and deep sequencing. Science 333, 1593-1602 (2011).

13. Doria-Rose, N. A. et al. Developmental pathway for potent V1V2-directed HIV-neutralizing antibodies. Nature 509, 55-62 (2014).

14. Gorman, J. et al. Structures of HIV-1 Env V1V2 with broadly neutralizing antibodies reveal commonalities that enable vaccine design. Nat. Struct. Mol. Biol. 23, 81-90 (2016).

15. McLellan, J. S. et al. Structure of HIV-1 gp120 V1/V2 domain with broadly neutralizing antibody PG9. Nature 480, 336-343 (2011).

16. Walker, L. M. et al. Broad and potent neutralizing antibodies from an African donor reveal a new HIV-1 vaccine target. Science 326, 285-289 (2009).

17. Andrabi, R. et al. Identification of common features in prototype broadly neutralizing antibodies to HIV envelope V2 apex to facilitate vaccine design. Immunity 43, 959-973 (2015).

18. Briney, B. S., Willis, J. R. \& Crowe, J. E. Jr. Human peripheral blood antibodies with long HCDR3s are established primarily at original recombination using a limited subset of germline genes. PLoS One 7, e36750 (2012)

19. Walker, L. M. et al. Broad neutralization coverage of HIV by multiple highly potent antibodies. Nature 477, 466-470 (2011).

20. Pejchal, R. et al. A potent and broad neutralizing antibody recognizes and penetrates the HIV glycan shield. Science 334, 1097-1103 (2011).

21. Kong, L. et al. Supersite of immune vulnerability on the glycosylated face of HIV-1 envelope glycoprotein gp120. Nat. Struct. Mol. Biol. 20, 796-803 (2013).

22. Garces, F. et al. Affinity maturation of a potent family of HIV antibodies is primarily focused on accommodating or avoiding glycans. Immunity $\mathbf{4 3}$ 1053-1063 (2015).

23. Huang, J. et al. Broad and potent neutralization of HIV-1 by a gp41-specific human antibody. Nature 491, 406-412 (2012)

24. Stiegler, G. et al. A potent cross-clade neutralizing human monoclonal antibody against a novel epitope on gp41 of human immunodeficiency virus type 1. AIDS Res. Hum. Retroviruses 17, 1757-1765 (2001).

25. Muster, T. et al. Cross-neutralizing activity against divergent human immunodeficiency virus type 1 isolates induced by the gp41 sequence ELDKWAS. J. Virol. 68, 4031-4034 (1994).

26. Muster, T. et al. A conserved neutralizing epitope on gp41 of human immunodeficiency virus type 1. J. Virol. 67, 6642-6647 (1993).

27. Ofek, G. et al. Structure and mechanistic analysis of the anti-human immunodeficiency virus type 1 antibody $2 \mathrm{~F} 5$ in complex with its gp41 epitope. J. Virol. 78, 10724-10737 (2004).

28. Ofek, G. et al. Relationship between antibody 2 F5 neutralization of HIVand hydrophobicity of its heavy chain third complementarity-determining region. J. Virol. 84, 2955-2962 (2010).

29. Irimia, A., Sarkar, A., Stanfield, R. L. \& Wilson, I. A. Crystallographic identification of lipid as an integral component of the epitope of hiv broadly neutralizing antibody 4E10. Immunity 44, 21-31 (2016).

30. Haynes, B. F., Moody, M. A., Verkoczy, L., Kelsoe, G. \& Alam, S. M. Antibody polyspecificity and neutralization of HIV-1: a hypothesis. Hum. Antibodies 14, 59-67 (2005).

31. Kong, R. et al. Fusion peptide of HIV-1 as a site of vulnerability to neutralizing antibody. Science 352, 828-833 (2016).

32. Carr, C. M. \& Kim, P. S. A spring-loaded mechanism for the conformational change of influenza hemagglutinin. Cell 73, 823-832 (1993).

33. van Gils, M. J. et al. An HIV-1 antibody from an elite neutralizer implicates the fusion peptide as a site of vulnerability. Nat. Microbiol. 2, 16199 (2016).

34. Blattner, C. et al. Structural delineation of a quaternary, cleavage-dependent epitope at the gp41-gp120 interface on intact HIV-1 Env trimers. Immunity 40, 669-680 (2014).

35. Boudko, S. P. et al. Crystal structure of human collagen XVIII trimerization domain: A novel collagen trimerization Fold. J. Mol. Biol. 392 787-802 (2009).
36. Tan, K. et al. Crystal structure of the TSP-1 type 1 repeats: a novel layered fold and its biological implication. J. Cell Biol. 159, 373-382 (2002).

37. Chu, R. et al. Redesign of a four-helix bundle protein by phage display coupled with proteolysis and structural characterization by NMR and X-ray crystallography. J. Mol. Biol. 323, 253-262 (2002).

38. Mougous, J. D. et al. A virulence locus of Pseudomonas aeruginosa encodes a protein secretion apparatus. Science 312, 1526-1530 (2006).

39. Harris, J. R. \& Markl, J. Keyhole limpet hemocyanin (KLH): a biomedical review. Micron 30, 597-623 (1999).

40. Sanders, R. W. et al. A next-generation cleaved, soluble HIV-1 Env trimer, BG505 SOSIP.664 gp140, expresses multiple epitopes for broadly neutralizing but not non-neutralizing antibodies. PLoS Pathog. 9, e1003618 (2013).

41. Kwon, Y. D. et al. Crystal structure, conformational fixation and entry-related interactions of mature ligand-free HIV-1 Env. Nat. Struct. Mol. Biol. 22, 522-531 (2015)

42. Trkola, A. et al. Human monoclonal antibody 2G12 defines a distinctive neutralization epitope on the gp120 glycoprotein of human immunodeficiency virus type 1. J. Virol. 70, 1100-1108 (1996).

43. Trkola, A. et al. Delay of HIV-1 rebound after cessation of antiretroviral therapy through passive transfer of human neutralizing antibodies. Nat. Med. 11, 615-622 (2005).

44. Trkola, A. et al. In vivo efficacy of human immunodeficiency virus neutralizing antibodies: estimates for protective titers. J. Virol. 82, 1591-1599 (2008)

45. Pauthner, M. et al. Elicitation of robust tier 2 neutralizing antibody responses in nonhuman primates by HIV envelope trimer immunization using optimized approaches. Immunity 46, 1073-1088.e6 (2017).

46. Sanders, R. W. et al. HIV-1 vaccines. HIV-1 neutralizing antibodies induced by native-like envelope trimers. Science 349, aac4223 (2015).

47. de Taeye, S. W. et al. Immunogenicity of stabilized HIV-1 envelope trimers with reduced exposure of non-neutralizing epitopes. Cell 163, 1702-1715 (2015)

48. Guenaga, J. et al. Structure-guided redesign increases the propensity of HIV Env to generate highly stable soluble trimers. J. Virol. 90, 2806-2817 (2015).

49. Kong, L. et al. Uncleaved prefusion-optimized gp140 trimers derived from analysis of HIV-1 envelope metastability. Nat. Commun. 7, 12040 (2016).

50. Zhou, T. et al. Quantification of the impact of the HIV-1-glycan shield on antibody elicitation. Cell Rep. 19, 719-732 (2017).

51. Ekiert, D. C. et al. Antibody recognition of a highly conserved influenza virus epitope. Science 324, 246-251 (2009).

52. Joyce, M. G. et al. Vaccine-induced antibodies that neutralize group 1 and group 2 influenza A viruses. Cell 166, 609-623 (2016).

53. Furuyama, W. et al. Discovery of an antibody for pan-Ebolavirus therapy. Sci. Rep. 6, 20514 (2016).

54. Wec, A. Z. et al. Antibodies from a human survivor define sites of vulnerability for broad protection against Ebolaviruses. Cell 169, 878-890.e15 (2017).

55 . Zhao, X. et al. Immunization-elicited broadly protective antibody reveals Ebolavirus fusion loop as a site of vulnerability. Cell 169, 891-904.e15 (2017).

56. Hastie, K. M. et al. Structural basis for antibody-mediated neutralization of Lassa virus. Science 356, 923-928 (2017).

57. Sok, D. et al. Rapid elicitation of broadly neutralizing antibodies to HIV by immunization in cows. Nature 548, 108-111 (2017).

58. McCoy, L. E. et al. Potent and broad neutralization of HIV-1 by a llama antibody elicited by immunization. J. Exp. Med. 209, 1091-1103 (2012)

59. Muyldermans, S. Nanobodies: natural single-domain antibodies. Annu. Rev. Biochem. 82, 775-797 (2013).

60. Wang, F. et al. Reshaping antibody diversity. Cell 153, 1379-1393 (2013).

\section{Acknowledgements}

We thank J. Chrzas, U. Chinte, Z. Jin, and staff at SER-CAT (Southeast Regional Collaborative Access Team) for help with X-ray diffraction data collection; B. DeKosky for suggestions on lineage discrimination, $\mathrm{W}$. Rice and SEMC staff for assistance with cryo-EM data collection; and J. Stuckey for assistance with figures. We thank D. Burton and M. Fineberg (International AIDS Vaccine Initiative (IAVI), Neutralizing Antibody Consortium (NAC)) for antibodies, including PGT122 used in cryo-EM studies, B. Haynes (The Duke Center for HIV/AIDS Vaccine Immunology and Immunogen Discovery (CHAVI-ID)) for information on antibody CH07, R. Sanders (Academisch Medisch Centrum Universiteit van Amsterdam (AMC)) for information on antibody ACS202, B. Graham (Vaccine Research Center, NIAID, NIH) for murine antibody 5C4, and the WCMC/AMC/TSRI HIVRAD team for their contributions to the design and validation of near-native mimicry for soluble BG505 SOSIP.664 trimers. We thank members of the Structural Biology Section, Structural Bioinformatics Core Section, and Human Immunology Section of the Vaccine Research Center for helpful comments. Support for this work was provided by the Intramural Research Program of the Vaccine Research Center, National Institute of Allergy and Infectious Diseases, National Institutes of Health. This work was also supported in part by IAVI's NAC (J.R.M and P.D.K.) and with federal funds from the Frederick National Laboratory for Cancer Research, NIH, under contract HHSN261200800001E (Y.T.). I.S.G. received support from NIH grant R01 AI131722. 
Some of this work was performed at the Simons Electron Microscopy Center and National Resource for Automated Molecular Microscopy, located at the New York Structural Biology Center, supported by grants from the Simons Foundation (SF349247) NYSTAR, and the NIH National Institute of General Medical Sciences (GM103310), with additional support from Agouron Institute (F00316) and NIH (OD019994). Use of insertion device 22 (SER-CAT) at the Advanced Photon Source was supported by the US Department of Energy, Basic Energy Sciences, Office of Science, under contract W-31-109-Eng-38.

\section{Author contributions}

K.X. conceived and led the project and determined crystal structures; P.A. determined cryo-EM structures; R.K. and N.A.D.-R. coordinated neutralization assessments; C.C. coordinated guinea pig and NHP immunization; G.-Y.C. coordinated statistical and bioinformatical analyses; K.L. prepared proteins and co-determined crystal structures; M.K.L. and R.T.B. assessed antibody neutralization in the large panel; C.-H.S. performed antibody sequence analyses; M.S. performed all Alanine-Glycine scans; B.Z. prepared antibodies for the large panel and performed various binding analyses; T.Z. performed SPR analyses, calculated the antibody's approach angle to the trimer, and made antibody neutralization dendrograms; M.A. performed antibody autoreactivity tests; I.S.G. performed antibody neutralization fingerprint analyses; T.L. performed molecular dynamics analyses; S.O'D., K.M., C.W.C., E.G.V., and A.P.R. co-performed neutralization assays; A.D., D.P., B.Z., and Y.Y. helped with protein expression; E.T.E., V.P.D., and H.W. helped with cryo-EM structures; X.C., H.G., J.G., M.S., and Y.D.K. performed protein purification; K.R.H., A.J.J., K.E.F., D.G.S., and J.-P.T. assisted in the NHP study; Y.-T.L. and Y.W. assisted with X-ray crystal dataset processing; B.Z., L.O., and M.C. helped with immunogen preparation and characterization; R.R. and S.K.F. performed statistical and bioinformatical analyses; Z.S. performed antibody gene comparisons; Y.T. performed negative-stain EM; T.Y.O. participated in immunogen binding tests; A.F.Z. helped with SPR assays; R.C. and L.Y. supervised the research team in GenScript; A.B.M. supervised MSD-based immunogen antigenicity characterization; L.S. supervised antibody gene analyses and comparison; B.C. and C.S.P. supervised cryo-EM studies; K.X., L.S.,

J.R.M., and P.D.K. wrote the manuscript; and all authors read, edited, and approved the manuscript. J.R.M. and P.D.K. conceived and supervised the study.

\section{Competing interests}

The authors declare no competing financial interests.

\section{Additional information}

Supplementary information is available for this paper at https://doi.org/10.1038/ s41591-018-0042-6.

Reprints and permissions information is available at www.nature.com/reprints. Correspondence and requests for materials should be addressed to J.R.M. or P.D.K. Publisher's note: Springer Nature remains neutral with regard to jurisdictional claims in published maps and institutional affiliations. 


\section{Methods}

Peptide synthesis and peptide-carrier protein conjugate preparation. HIV-1 fusion peptides (FPs) were synthesized (GenScript) with a free amine at the $\mathrm{N}$ terminus. FP His-tagged peptides were synthesized (GenScript) with a six-histidine-residue tag at the $\mathrm{C}$ terminus. To prepare peptide-carrier protein conjugates, each peptide with a cysteine residue appended to the $\mathrm{C}$ terminus was conjugated to lysine residuse on the carrier protein keyhole limpet hemocyanin (KLH; Thermo-Scientific) using m-maleimidobenzoyl- $N$-hydroxysuccinimide ester (MBS), following the manufacturer's protocol.

Protein expression and purification. BG505 SOSIP, BG505 DS-SOSIP, and their glycan-deficient variants were expressed and purified as described previously ${ }^{41}$. FP-epitope scaffold proteins, including FP-1M6T, FP-1Y12, FP-3HSH, and FP-1SLF were expressed and purified as described previously ${ }^{31}$. vFP1.01, vFP7.04, vFP16.02, and vFP20.01 antibodies used for structure determination were prepared as below. Heavy chain plasmids, encoding the chimera of mouse variable domain and human constant domain, with HRV3C cleavage site in the hinge region; and light chain plasmids, encoding the chimera of mouse variable domain and human constant domain, were co-transfected in Expi293F cells (Thermo Fisher) using Turbo293 transfection reagent (SPEED BioSystem) according to the manufacturer's protocol. Transfected cells were incubated in shaker incubators at $120 \mathrm{rpm}, 37^{\circ} \mathrm{C}$, $9 \% \mathrm{CO}_{2}$ overnight. On the second day, one-tenth culture volume of AbBooster medium (ABI scientific) was added to each flask of transfected cells and cell cultures were incubated at $120 \mathrm{rpm}, 33^{\circ} \mathrm{C}, 9 \% \mathrm{CO}_{2}$ for an additional $5 \mathrm{~d}$. Cell culture supernatants were harvested $6 \mathrm{~d}$ post-transfection. IgGs were purified from the supernatants using protein A chromatography: after PBS wash and low $\mathrm{pH}$ glycine buffer elution, the eluate was immediately neutralized using $10 \%$ volume of $1 \mathrm{M}$ Tris buffer, $\mathrm{pH}$ 8.0. Fabs were obtained either by HRV3C cleavage or papain digestion. The fragmented Fabs were further purified by size-exclusion chromatograph (SEC) in a Superdex 200 column (GE) with a buffer containing $5 \mathrm{mM}$ HEPES, pH 7.5, and $150 \mathrm{mM} \mathrm{NaCl}$.

Negative-stain electron microscopy. Samples were diluted with a buffer containing $20 \mathrm{mM}$ HEPES, pH 7.0, and $150 \mathrm{mM} \mathrm{NaCl}$, adsorbed to a freshly glow-discharged carbon-film grid, washed with the above buffer, and stained with $0.7 \%$ uranyl formate. Images were collected semiautomatically at a magnification of $100,000 \times$ using SerialEM ${ }^{61}$ on a FEI Tecnai T20 microscope equipped with a $2 \mathrm{k} \times 2 \mathrm{k}$ Eagle CCD camera and operated at $200 \mathrm{kV}$. The pixel size was $0.22 \mathrm{~nm} /$ pixel. Particles were picked manually using the swarm mode in e2boxer from the EMAN2 software package ${ }^{62}$. Reference-free 2D classification was performed using EMAN2 and SPIDER ${ }^{63}$

\section{Antigenic characteristics of fusion peptide immunogens with Biolayer} Interferometry and MSD-ECLIA. Antigenic characteristics of KLH-coupled fusion peptide, FP scaffolds, and BG505 Env trimers to various FP-targeting and non-FP-targeting HIV antibodies were assessed with Biolayer Interferometry on an Octet RED384 (ForteBio) instrument and with MSD-ECLIA as previously described ${ }^{41}$.

\section{Antigenicity score. Antigenicity scores for the FP immunogens were calculated} according to the metric defined in Supplementary Fig. 1a. This metric sums the average binding of neutralizing antibodies versus poorly/non-neutralizing antibodies, with binding defined as a function of the logarithm of antigen-binding affinity relative to upper and lower binding limits. This summation is weighted by the site targeted. An antigenicity score of 1 for an FP immunogen therefore indicates both high specificity and tight affinity for FP-directed neutralizing antibodies; an antigenicity score of 0 for an FP immunogen would indicate either low specificity or weak affinity for FP-directed neutralizing antibodies. In Fig. 1a, the antigenicity score was calculated with only FP-directed antibodies; we anticipate that the FP-antigenicity score for trimer immunogens would decease relative to FP-specific immunogens, such as FP8-KLH, if non-FP-directed antibodies were considered.

Mouse immunization (GenScript). Female mice (C57BL/6) around 8 weeks old were immunized in 2-week intervals with either HIV-1 Env trimer or FP-KLH, using either Adjuplex as adjuvant (Sigma-Aldrich Inc., MO) for trimer or GS-adjuvant (GenScript) for FP-KLH. We used $50 \mu \mathrm{g}$ of immunogens for prime immunization and $25 \mu \mathrm{g}$ immunogens in boost immunization. Intraperitoneal (IP) injections were used for all mouse immunizations. Sera samples were collected either $7 \mathrm{~d}$ or $14 \mathrm{~d}$ after each immunization for ELISA and other analyses.

All experiments were performed in accordance with protocols reviewed and approved by GenScript's Institutional Animal Care and Use Committee (IACUC, \#ANT14-003 and \#ANT17-003). All mice were housed and cared for in a GenScript facility accredited by the Association for Assessment and Accreditation of Laboratory Animal Care International (AAALAC International).

Hybridoma creation and monoclonal antibody production. Terminal boosts were performed on the top responders from each immunization scheme, as assessed with ELISA, against the immunogens 3 weeks after the last immunization.
Mice spleens were harvested $3 \mathrm{~d}$ after the terminal boost, and hybridomas were generated for monoclonal antibody selection following the standard procedure at GenScript. Monoclonal antibody selection was based on affinity to FP-scaffold (FP-1M6T) and BG505 Env trimer as measured by ELISA.

Guinea pig and NHP protocols and immunizations. For immunization studies, all animals were housed and cared for in accordance with local, state, federal, and institute policies in an American Association for Accreditation of Laboratory Animal Care-accredited facility at the Vaccine Research Center, NIAID, NIH, or at a contract facility (Bioqual Inc., MD). All animal experiments were reviewed and approved by the Animal Care and Use Committee of the Vaccine Research Center, NIAID, NIH, and covered under protocols VRC-13-431, VRC16-552, and VRC-16-667.

Female Hartley guinea pigs with body weights of $300 \mathrm{~g}$ each were purchased from Charles River Laboratories, MA. For each immunization, $400 \mu \mathrm{L}$ of immunogen mix, containing $25 \mu \mathrm{g}$ of specified, filter-sterilized protein immunogen and $80 \mu \mathrm{L}$ of Adjuplex (Sigma-Aldrich Inc., MO, or Adjuplex equivalent formulated at VRC based on US patent No. 6,676,958 B2, Example 1) in PBS, was injected into the muscle of the two hind legs. While animals were under anesthesia, blood was collected through retro-orbital bleeding for serological analyses.

Female and male Indian-origin rhesus macaques with body weights of $2-9 \mathrm{~kg}$ were used for immunization studies. For each immunization, $1 \mathrm{~mL}$ of immunogen mix, containing $100 \mu \mathrm{g}$ of specified, filter-sterilized protein immunogen and $200 \mu \mathrm{L}$ of Adjuplex (Sigma-Aldrich Inc., MO, or Adjuplex equivalent formulated at VRC based on US patent No. 6,676,958 B2, Example 1) in PBS, was injected via a needle syringe into the caudal thighs of the two hind legs. Blood was collected 2 weeks postimmunization for serological analyses.

ELISA. Fusion peptide ELISAs: Costar High Binding Half-Area 96-well plates (Corning, Kennebunk, ME) were coated with $50 \mu \mathrm{L} /$ well of $2 \mu \mathrm{g} / \mathrm{mL}$ scaffold proteins in PBS overnight at $4{ }^{\circ} \mathrm{C}$. Between steps, plates were washed five times with PBS-T (PBS $+0.05 \%$ Tween) and incubated at $37^{\circ} \mathrm{C}$ for $1 \mathrm{~h}$. After coating, plates were blocked with $100 \mu \mathrm{L} /$ well of blocking buffer (B3T: $150 \mathrm{mM} \mathrm{NaCl}$, $50 \mathrm{mM}$ Tris-HCl, $1 \mathrm{mM}$ EDTA, 3.3\% FBS, $2 \%$ bovine albumin, $0.07 \%$ Tween $20,0.02 \%$ thimerosal). Twofold serial dilution of 1:100 preimmunization and $1: 1,000$ postimmunization sera were used in the ELISA. Goat anti-mouse IgG (HRP-conjugated, GenScript) at 1:5,000 dilution was used for detection. Plates were developed with tetramethylbenzidine (TMB) substrate (SureBlue, KPL, Gaithersburg, MD) for 10 min before adding $1 \mathrm{~N}$ sulfuric acid (Fisher Chemical) to stop the reaction. Plates were read at $450 \mathrm{~nm}$ (Molecular Devices, SpectraMax using SoftMax Pro 5 software) and the optical densities (OD) were recorded.

BG505 SOSIP D7324 capture ELISAs: Costar High Binding Half-Area 96-well plates (Corning, Kennebunk, ME) were coated with $50 \mu \mathrm{L} /$ well of $2 \mu \mathrm{g} / \mathrm{mL}$ of sheep D7324 antibody (AALTO Bio Reagents) in PBS overnight at $4{ }^{\circ} \mathrm{C}$. Between steps, except for addition of trimer, plates were washed five times with PBS-T (PBS $+0.05 \%$ Tween) and incubated at room temperature (RT; $\left.20-25^{\circ} \mathrm{C}\right)$ for $1 \mathrm{~h}$ After coating, plates were blocked with $100 \mu \mathrm{L} /$ well of blocking buffer (5\% skim milk, $2 \%$ bovine albumin, $0.1 \%$ Tween 20 in TBS). Next, $50 \mu \mathrm{L} /$ well of $0.5 \mu \mathrm{g} / \mathrm{mL}$ D7324-tagged BG505.SOSIP trimer diluted in 10\% FBS in PBS was added and incubated at RT for $2 \mathrm{~h}$. Twofold serial dilution of 1:100 preimmunization and 1:1,000 postimmunization sera were used in the ELISA. Goat anti-mouse IgG (HRP-conjugated, GenScript) at 1:5,000 dilution was used for detection. Plates were developed with tetramethylbenzidine (TMB) substrate (SureBlue, KPL, Gaithersburg, MD) for 10 min before adding $1 \mathrm{~N}$ sulfuric acid (Fisher Chemical) to stop the reaction. Plates were read at $450 \mathrm{~nm}$ (Molecular Devices, SpectraMax using SoftMax Pro 5 software) and the optical densities (OD) were recorded. ELISA responses were plotted using PRISM (PRISM 7 GraphPad Software for Mac OS X).

Genetic assignment of antibodies. Antibody sequences were submitted to the ImMunoGeneTics information system (IMGT, http://www.imgt.org), subjected to variable $(\mathrm{V})$, diverse $(\mathrm{D})$, and joining $(\mathrm{J})$ gene identification by alignment with the mouse germline sequences of the IMGT reference directory, and subject to IMGT/ JunctionAnalysis for a detailed analysis of the V-J and V-D-J junctions. We only considered confirmed functional germline genes for the assigned germline. Clustal Omega software was used to prepare multiple-sequence alignments of antibody sequences for maximum-likelihood phylogenetic tree construction using the DNAML program in the PHYLIP package version 3.69 (http://evolution.genetics. washington.edu/phylip.html). Calculations were performed based on empirical base frequencies with transition/transversion $(\mathrm{Ti} / \mathrm{Tv})$ ratio of 2.0. Dendroscope 3 (dendroscope.org) was used to visualize phylogenetic trees ${ }^{64}$. The amino acid sequence alignments were visualized using BioEdit v7.2.5 editing software ${ }^{65}$. To calculate the minimum number of mutations required to switch between two different unmutated common ancestors, the unmutated common ancestor sequence was prepared by reverting the assigned $\mathrm{V}(\mathrm{D}) \mathrm{J}$ gene sequences into their corresponding germline sequences. Differences between unmutated common ancestor sequences were counted as the minimal mutation number required to switch from one unmutated common ancestor to another. 
Antibody alanine/glycine scan. Binding of the vaccine elicited mouse vFP antibodies to 16 His-tagged fusion peptide (residues 512-521), including wildtype and alanine/glycine mutants, was assessed using a fortéBio Octet Red384 instrument. Briefly, the 16 peptides at $50 \mu \mathrm{g} / \mathrm{mL}$ in PBS were loaded onto Ni-NTA biosensors using their C-terminal histidine tags for $60 \mathrm{~s}$. Typical capture levels were between 1.1 and $1.3 \mathrm{~nm}$, and variability within a row of eight tips did not exceed $0.1 \mathrm{~nm}$. These peptide-bound biosensors were equilibrated in PBS for $60 \mathrm{~s}$ followed by capture of the antigen binding fragments (Fabs, $250 \mathrm{nM}$ ) of the vaccine elicited vFP antibodies, VRC34.01, and an RSV F antibody (motavizumab) for $120 \mathrm{~s}$ and a subsequent dissociation step in PBS

In all Octet measurements, parallel correction to subtract systematic baseline drift was carried out by subtracting the measurements recorded for a loaded sensor incubated in PBS. Data analysis was carried out using Octet software, version 9.0. The normalized responses obtained from one dataset or from a triplicate dataset were plotted using PRISM (PRISM 7 GraphPad software).

Surface plasmon resonance assay. Binding affinities and kinetics of antibodies to HIV-1 DS-SOSIP trimers and His-tagged fusion peptide were assessed by surface plasmon resonance on a Biacore T-200 (GE Healthcare) at $25^{\circ} \mathrm{C}$. To test antibody binding with HIV-1 DS-SOSIP trimers, $2 \mathrm{G} 12 \mathrm{IgG}$ was first immobilized on flow cells of a CM5 chip at $\sim 3,000-8,000$ response units. BG505 DS-SOSIP trimer and its glycan-deleted mutants, BG505 DS-SOSIP. $\Delta 88$ and BG505 DS-SOSIP. $\Delta 611$, at $500 \mathrm{nM}$ in HBS-EP+ buffer (10 mM HEPES, pH 7.4, $150 \mathrm{mM} \mathrm{NaCl}, 3 \mathrm{mM}$ EDTA and $0.05 \%$ surfactant $\mathrm{P}-20$ ) were then captured onto the $2 \mathrm{G} 12$ of one flow cell by flowing the protein solution over it for $60 \mathrm{~s}$ at a rate of $6 \mu \mathrm{L} / \mathrm{min}$. Binding affinities for the 2G12-captured trimer were determined using a serial dilution of antibody Fab solutions, starting at $200 \mathrm{nM}$ during the association phase. A dissociation phase at $30 \mu \mathrm{L} / \mathrm{min}$ for $300 \mathrm{~s}$ was used to determine binding kinetics. The surface was regenerated by flowing $3 \mathrm{M} \mathrm{MgCl}_{2}$ solution over it for $30 \mathrm{~s}$ at a flow rate of $50 \mu \mathrm{L} / \mathrm{min}$. Blank sensorgrams were obtained injecting the same volume of HBS-EP+ buffer in place of the antibody Fab solution. Sensorgrams of the concentration series were corrected with corresponding blank curves and fitted globally with Biacore T200 evaluation software using a 1:1 Langmuir model of binding.

Affinity of antibody Fab to the His-tagged fusion peptide was measured on a Ni-NTA sensor chip (GE Healthcare). The Ni-NTA surface was activated by injection of $5 \mathrm{mM}$ of $\mathrm{Ni}_{2} \mathrm{SO}_{4}$ in HBS-P+ buffer (10 mM HEPES, pH 7.4, $150 \mathrm{mM}$ $\mathrm{NaCl}$, and $0.05 \%$ surfactant $\mathrm{P}-20$ ) for $60 \mathrm{~s}$ at $6 \mu \mathrm{L} / \mathrm{min}$ and then stabilized by washing with HBS-EP+ buffer containing $3 \mathrm{mM}$ EDTA for $60 \mathrm{~s}$ at $30 \mu \mathrm{L} / \mathrm{min}$. Fusion peptide with His-tag at $20 \mathrm{ng} / \mathrm{mL}$ was captured at $6 \mu \mathrm{L} / \mathrm{min}$ flow rate for $60 \mathrm{~s}$ over the nickel-activated sensor surface. Binding affinities for the 2G12captured trimer were determined using a serial dilution of antibody Fab solutions starting at $200 \mathrm{nM}$ during the association phase. A dissociation phase at $30 \mu \mathrm{L} / \mathrm{min}$ for $300 \mathrm{~s}$ was used to determine binding kinetics. The surface was regenerated by flowing $300 \mathrm{mM}$ imidazole to both channels at $6 \mu \mathrm{L} / \mathrm{min}$ for $60 \mathrm{~s}$. Sensorgrams of the concentration series were corrected with corresponding blank curves and fitted globally with Biacore T200 evaluation software using a 1:1 Langmuir model of binding.

HIV-1 Env mutagenesis. Site-directed mutagenesis on HIV-1 Env plasmids was performed through GeneImmune Biotechnology LLC, NY. T90A and S613A mutations were created to remove glycans 88 and 611, respectively.

HIV-1 Env-pseudotyped virus. 293T-grown HIV-1 Env-pseudotyped virus stocks were generated by cotransfection of the wild-type or mutant Env expression plasmids with a pSG3 $\Delta$ Env backbone ${ }^{11}$.

Neutralization assays. A single round of entry neutralization assays using TZM-bl target cells were performed to assess monoclonal antibody neutralization as described ${ }^{11}$. Briefly, the monoclonal antibodies were tested via fivefold serial dilutions starting at up to $500 \mu \mathrm{g} / \mathrm{mL}$. Monoclonal antibodies were mixed with the virus stocks in a total volume of $50 \mu \mathrm{L}$ and incubated at $37^{\circ} \mathrm{C}$ for $1 \mathrm{~h}$. We then added $20 \mu \mathrm{L}$ of TZM-bl cells $(0.5 \mathrm{million} / \mathrm{mL})$ to the mixture and incubated it at $37^{\circ} \mathrm{C}$ overnight. On day $2,130 \mu \mathrm{L}$ cDMEM were added, and cells were lysed on day 3 and assessed for luciferase activity (RLU). The $50 \%$ and $80 \%$ inhibitory concentrations $\left(\mathrm{IC}_{50}\right.$ and $\mathrm{IC}_{80}$ ) were determined using a hill-slope regression analysis as described ${ }^{11}$.

To assess monoclonal antibody neutralization on a panel of $208 \mathrm{HIV}-1$ Envpseudotyped viruses, an automated 384-well microneutralization assay was performed as described previously ${ }^{66}$

Serum neutralization was also assessed in the single round of entry neutralization assays using TZM-bl target cells, as described above. Before evaluation, all sera from immunized and control animal were heat-inactivated at $56^{\circ} \mathrm{C}$ for $1 \mathrm{~h}$. All sera were tested via fourfold serial dilutions starting at 1:20 dilution.

Serum neutralization with FP competition was performed with serum in presence of either PEGylated FP9 (AVGIGAVFL) or PEGylated noncognate FLAG peptide. Mean and s.d. of results from triplicated experiments were determined.

Protein complex preparation. Antibody Fab and fusion peptide (residues 512-518) complexes were prepared by first dissolving fusion peptide in $100 \%$ DMSO at
$50 \mathrm{mg} / \mathrm{mL}$ concentration, then mixing it with Fab solution in a 10:1 molar ratio to reach a final protein complex centration of $15 \mathrm{mg} / \mathrm{mL}$.

Protein crystal screening. Antibody Fab and fusion peptide (residues 512-518) complexes were screened for crystallization in JCSG1-4 protein-crystal screening kits using a Cartesian Honeybee crystallization robot, as described previously ${ }^{15}$, and a mosquito robot. Crystals initially observed from the wells were manually reproduced. The vFP1.01/FP complex crystal grew in $0.2 \mathrm{M} \mathrm{AmSO}_{4}$ and $0.1 \mathrm{M}$ $\mathrm{NaOAc}, \mathrm{pH} 4.6$; the vFP7.04/FP complex crystal grew in $0.1 \mathrm{M}$ MES, $\mathrm{pH}$ 6.0, 30\% PEG 6000; the vFP16.02/FP complex crystal grew in $0.1 \mathrm{M} \mathrm{NaOAc}, \mathrm{pH} 4.5$, and 2 $\mathrm{M} \mathrm{AmSO}_{4}$; the vFP20.01/FP complex crystal grew in $0.1 \mathrm{M}$ citric acid, $\mathrm{pH} 3.5$, ad 2 $\mathrm{M} \mathrm{AmSO}_{4}$; and the vFP5.01/FP complex crystals grew in $0.2 \mathrm{M} \mathrm{MgCl}_{2}, 0.1 \mathrm{M}$ Tris$\mathrm{HCl}, \mathrm{pH} 8.5$, and $20 \%$ PEG 8000 .

$\mathrm{X}$-ray data collection, structure solution, model building, and refinement. Crystals were cryoprotected in $20 \%$ glycerol and flash-frozen in liquid nitrogen. Data were collected at a temperature of $100 \mathrm{~K}$ and a wavelength of $1.00 \AA$ at the SER-CAT beamline ID-22 (Advanced Photon Source, Argonne National Laboratory). Diffraction data were processed with the HKL2000 suite ${ }^{67}$. Structure solution was obtained by molecular replacement with Phaser using homologous Fab structures (PDB IDs: 3BKY for vFP1-class antibody complex and 3LEY for vFP5.01 antibody complex) as search models. Model building was carried out with $\operatorname{Coot}^{68}$. Refinement was carried out with Phenix ${ }^{69}$. Ramachandran statistical analysis indicated that the final structures contained no disallowed residues or no more than $0.23 \%$ disallowed residues. Data collection and refinement statistics are shown in Supplementary Table 4.

Cryo-EM data collection and processing. The Env trimer used in cryo-EM was generated in the GNTI- cell line as described previously ${ }^{41}$. To prepare Env complexes, BG505 DS-SOSIP at a final concentration of $0.3-0.5 \mathrm{mg} / \mathrm{mL}$ was incubated with four- to fivefold molar excess of the antibody Fab fragments for 30-60 min. To prevent aggregation during vitrification, the sample was incubated in $0.085 \mathrm{mM} n$-dodecyl $\beta$-D-maltoside (DDM). The vFP1.01 and vFP5.01 bound complexes were vitrified by applying $3 \mu \mathrm{L}$ of sample to freshly plasma-cleaned C-flat holey carbon grids (CF-1.2/1.3-4C; EMS, Hatfield, PA) for vFP1.01 and gold grids for vFP5.01, allowing the sample to adsorb to the grid for $60 \mathrm{~s}$, followed by blotting with filter paper and plunge-freezing into liquid ethane using the CP3 cryo-plunger (Gatan, Inc.; $20^{\circ} \mathrm{C}, 85-90 \%$ relative humidity)

The vFP16.02 and vFP20.01 bound complexes were vitrified using a semiautomated Spotiton V1.0 robot $^{70,71}$ The grids used were specially designed Nanowire self-blotting grids with a carbon lacey supporting substrate ${ }^{72}$. Sample was dispensed onto these nanowire grids using a picoliter piezo dispensing head. A total of $\sim 5 \mathrm{~nL}$ sample was dispensed in a stripe across each grid, followed by a pause of a few milliseconds before the grid was plunged into liquid ethane.

Data were acquired using the Leginon system installed on a Titan Krios electron microscopes operating at $300 \mathrm{kV}$ and fitted with a Gatan K2 Summit direct detection device. The dose was fractionated over 50 raw frames and collected over a 10-s exposure time. Individual frames were aligned and dose-weighted using MotionCor $2^{73}$

CTF was estimated using the GCTF package ${ }^{74}$. Particles were picked using DoG Picker within the Appion pipeline ${ }^{75} .2 \mathrm{D}$ and $3 \mathrm{D}$ classifications were performed using RELION ${ }^{76}$. A map of unliganded BG505 SOSIP.664 (EMDB ID EMD-5782), lowpass-filtered to $60 \AA$, was used as the starting point of $3 \mathrm{D}$ classification, followed by $3 \mathrm{D}$ refinement in either RELION or cryoSparc ${ }^{77}$. For the vFP16.02 and vFP20.01 complexes, after 3D classification in RELION, an additional step of ab initio reconstruction was performed using cryoSparc.

Cryo-EM model fitting. Fits of HIV-1 trimer and Fab to the cryo-EM reconstructed maps were performed using Chimera ${ }^{78}$. Glycosylated BG505 SOSIP trimer structure (PDB ID: 5FYL) was used for the trimer fits. For antibody fitting, we used the fusion peptide-bound coordinates of vFP1.01 and vFP5.01. For the antibody Fabs, both orientations (rotated $\sim 180^{\circ}$ about the Fab longitudinal axis) were tested, and the optimal fit was decided based on map-to-model correlation and positioning of the fusion peptide bound to the Fab relative to Env. For the vFP16.02 and vFP20.01 bound complexes, the coordinates were further fit to the electron density by an iterative process of manual fitting using $\operatorname{Coot}^{68}$ and real space refinement within Phenix ${ }^{69}$. Molprobity ${ }^{79}$ and EMRinger ${ }^{80}$ were used to check geometry and evaluate structures at each iteration step. Figs. were generated in UCSF Chimera and PyMOL. Map-fitting cross correlations were calculated using the Fit-in-Map feature in UCSF Chimera. Map-to-model FSC curves were generated using EMAN2. Local resolution of cryo-EM maps was determined using RELION.

Defining vFP1-class antibody V-gene sequence signature. The V-gene sequence signatures for vFP1 class antibodies were defined by examining the vFP1 class antibody sequences that neutralize at least seven of the ten tested strains and the structures of FP in complex with vFP1.01, vFP16.02, and vFP20.01. A residue position was considered part of the sequence signature if at least one side-chain heavy atom was within $5 \AA$ of any fusion peptide heavy atom for all 
three complex structures, no more than three similar amino acid types had a combined prevalence of more than $90 \%$, and each of these amino acid types had a prevalence of more than $10 \%$.

Molecular dynamics simulation of mannose-5 Env trimer. By using the BG505 SOSIP.664 Env trimer structure (PDB ID: 4TVP) as a starting template, we modeled in a fully extended mannose- 5 moiety at each $N$-linked glycosylation sequon. The fusion peptide structure was then grafted onto our full mannose- 5 model followed by 5,000 steps of conjugate gradient energy minimization in implicit solvent using NAMD. The obtained structure was then solvated in a $17-\AA$ padding water box, neutralized by the addition of $\mathrm{NaCl}$ at a concentration of $150 \mathrm{mM}$. The CHARMM force field (https://www.charmm.org) was used to parameterize the protein (including CMAP corrections and mannose-9). TIP3P water parameterization ${ }^{81}$ was used to describe the water molecules.

Two independent molecular simulations were carried out using ACEMD molecular dynamics software on their METROCUBO workstation (https://www. acellera.com/products/GPU-hardware-molecular-dynamics-metrocubo/). The system was minimized for 2,000 steps, followed by equilibration using the NPT ensemble for $50 \mathrm{~ns}$ at $1 \mathrm{~atm}$ and $300 \mathrm{~K}$ using a timestep of $2 \mathrm{fs}$. We also used rigid bonds and a 9-A cutoff using PME for long range electrostatics. During the equilibration phase, heavy atoms on the protein were constrained by a $1 \mathrm{kcal} / \mathrm{mol}^{2}$ spring constant and slowly relaxed over the first $5 \mathrm{~ns}$. Following the relaxation phase, the protein was allowed to move freely and simulated for $500 \mathrm{~ns}$ under the NVT ensemble using ACEMD's NVT ensemble with a Langevin thermostat. To achieve a timestep of $4 \mathrm{ps}$, we used damping at $0.1 \mathrm{ps}-1$ and a hydrogen mass repartitioning scheme. Each simulation ran up to $500 \mathrm{~ns}$.

Conformations of the fusion peptide (residues 512-519) were extracted from the MD simulations every 100 ps, producing an ensemble of 30,000 structures. Prody (http://prody.csb.pitt.edu) was used to perform the principal component analysis of backbone atoms. The conformations of five crystalized fusion peptides were then projected into the eigenspace defined by the first two components: vFP1.01, vF5.01, PGT151 (PDB: 5FUU), VRC34.01 (PDB: 5I8H) and clade G (PDB: 5FYJ).

Analysis of antibody angle of approach to HIV-1 Env. To compare modes of antibody recognition of HIV-1 Env by vaccine-elicited fusion peptide antibodies and VRC34.01, structural models of antibody in complex with HIV-1 BG505 SOSIP Env trimer derived from X-ray crystallography and EM were superimposed by pairwise alignment of the C $\alpha$ atom coordinates of the HIV-1 Env. To simplify the comparison, we first defined two common axes on the HIV-1 Env, the trimer axis and the protomer axis, as reference lines. The trimer axis was defined by two points, each with $x, y, z$ coordinates obtained by averaging the coordinates of the $\mathrm{C} \alpha$ atom of a residue and its threefold symmetry mates on the same trimer. The protomer axis was defined by a line perpendicular to the trimer axis and that passes through the center of the protomer. On the antibody side, we also defined two axes for each Fab. The long axis of a Fab was defined by two points, one point from the variable domain with $x, y, z$ coordinates obtained by averaging the coordinates of the C $\alpha$ atom of the four conserved Cys (Cys 22 and Cys 92 of heavy chain, and Cys 23 and Cys88 of light chain), and the other point from the constant domain with $x, y, z$ coordinates obtained by averaging the coordinates of the $\mathrm{C} \alpha$ atom of the four conserved Cys (Cys 140 and Cys196 of heavy chain, and Cys134 and Cys194 of light chain). The short axis of a Fab was defined by a line connecting $\mathrm{C} \alpha$ atoms of heavy chain Cys22 and light chain Cys23. Approaching angles of each antibody were then calculated as (i) angle between trimer axis and Fab long axis, and (ii) angle between the axis of major interacting protomer and Fab long axis. In addition, the angles between Fab variable domains were defined by the angles of their short axes. The axes were visualized in PyMOL by placing their coordinates in a PDB file.

Autoreactivity assay. Antibodies were assessed for autoreactivity by testing for binding to HEp2 cells by indirect immunofluorescence (Zeus Scientific, ANA HEp2 test system) and cardiolipin by ELISA (Inova Diagnostics, QUANTA Lite ACA IgG III), per the manufacturers' instructions. On HEp2 cells, antibodies were assigned a score between 0 and $3+$, using control antibodies as reference. In the cardiolipin binding assay, OD values were converted to GPLs using standard samples provided in the kit. Monoclonal antibodies that scored greater than 20 GPLs at $33 \mu \mathrm{g} / \mathrm{mL}$ were considered autoreactive.

Neutralization fingerprinting analysis. The neutralization fingerprint of a monoclonal antibody or polyclonal plasma is defined as the potency pattern with which the antibody/plasma neutralizes a set of diverse viral strains. The neutralization fingerprints of a set of monoclonal antibodies and NHP plasma were compared and clustered according to fingerprint similarity, as described previously ${ }^{82}$. A set of 132 strains $^{83}$ was used in the neutralization fingerprint analysis for Supplementary Fig. 11, and the 58 FP-selected strains were used for Fig. 6.

Associations between glycosylation patterns versus neutralization. We calculated the associations between the sequence variability of FP-neighboring glycosylation sites (HXB2 numbering 88, 241, 448, and 611) and large-panel neutralization using an approach implemented with R package SeqFeatR (https:// seqfeatr.zmb.uni-due.de). Briefly, for each residue position we created contingency table based on glycan occurrence and neutralization sensitivity and evaluated the association using Fisher's exact test. The resulting $P$ values were corrected using Holm's multiple testing method.

Statistical analysis. The correlation between neutralization breadth on the 58-strain panel with FP8 = AVGIGAVF and neutralization breadth on the full 208-strain panel was performed using Pearson correlation. The differences in number of neutralized strains between the FP-KLH-primed + DS-SOSIPboosted group and the DS-SOSIP-alone group were evaluated using one-tailed Mann-Whitney tests. The differences in neutralization between FP-KLH groups and the KLH-only group were evaluated using one-tailed Mann-Whitney tests. The correlation between antibody trimer binding and antibody neutralization breadth was performed using Pearson correlation. The differences in divergence of germline between antibodies from trimer-primed groups and antibodies from non-trimer-primed groups were evaluated using two-tailed Mann-Whitney tests. The differences in sera neutralization between trimer-boosted animals and nontrimer-boosted animals were evaluated using one-tailed Mann-Whitney tests. The association of FP-proximal N-glycan sequons (N88, N241, N448, and N611) and neutralization based on the 208-strain panel was evaluated using two-tailed Fisher's exact tests. Unless otherwise indicated, all data were plotted and graphed using GraphPad Prism, version 7.0; and $P<0.05$ indicated a statistically significant difference.

Reporting Summary. Further information on experimental design is available in the Nature Research Reporting Summary linked to this article.

Code availability. No studies deemed central to the conclusions were carried out with custom code.

Data availability. All data generated or analyzed during this study are included in this published article (and its Supplementary Information files). Atomic coordinates and structure factors of the reported crystal structures were deposited in the Protein Data Bank with PDB IDs 5TKJ, 5TKK, 6CDM, 6CDO, 6CDP. CryoEM reconstructions were deposited in the Electron Microscopy Data Bank with EMDB accession codes EMD-7460, EMD-7459, EMD-8420, EMD-8421, and EMD-8422, and in Protein Data Bank with PDB ID 6CDI and 6CDE. Heavy chainand light chain-variable sequences of monoclonal antibodies vFP1.01-vFP7.05 and vFP7.06-vFP 32.07 were deposited with GenBank under accession numbers KX949064-KX949087 and MH017667-MH017826, respectively.

\section{References}

61. Mastronarde, D. N. Automated electron microscope tomography using robust prediction of specimen movements. J. Struct. Biol. 152, 36-51 (2005).

62. Tang, G. et al. EMAN2: an extensible image processing suite for electron microscopy. J. Struct. Biol. 157, 38-46 (2007).

63. Frank, J. et al. SPIDER and WEB: processing and visualization of images in 3D electron microscopy and related fields. J. Struct. Biol. 116, 190-199 (1996)

64. Huson, D. H. et al. Dendroscope: an interactive viewer for large phylogenetic trees. BMC Bioinformatics 8, 460 (2007).

65. Hall, T. BioEdit: a user-friendly biological sequence alignment editor and analysis program for Windows 95/98/NT. Nucleic Acids Symposium Series 41, 95-98 (1999).

66. Sarzotti-Kelsoe, M. et al. Optimization and validation of the TZM-bl assay for standardized assessments of neutralizing antibodies against HIV-1. J. Immunol. Methods 409, 131-146 (2014).

67. Otwinowski, Z. \& Minor, W. Processing of X-ray diffraction data collected in oscillation mode. Methods Enzymol. 276, 307-326 (1997).

68. Emsley, P. \& Cowtan, K. Coot: model-building tools for molecular graphics. Acta Crystallogr. D Biol. Crystallogr. 60, 2126-2132 (2004).

69. Adams, P. D. et al. Recent developments in the PHENIX software for automated crystallographic structure determination. J. Synchrotron Radiat. 11, 53-55 (2004).

70. Razinkov, I. et al. A new method for vitrifying samples for cryoEM. J. Struct. Biol. 195, 190-198 (2016).

71. Dandey, V. P. et al. Spotiton: New features and applications. J. Struct. Biol. 202, 161-169 (2018)

72. Wei, H. et al. Optimizing "self-wicking" nanowire grids. J. Struct. Biol. 202, 170-174 (2018).

73. Zheng, S.Q. et al. MotionCor2: anisotropic correction of beam-induced motion for improved cryo-electron microscopy. Nat. Methods 14, 331-332 (2017)

74. Zhang, K. Gctf: real-time CTF determination and correction. J. Struct. Biol. 193, 1-12 (2016).

75. Lander, G. C. et al. Appion: an integrated, database-driven pipeline to facilitate EM image processing. J. Struct. Biol. 166, 95-102 (2009). 
76. Scheres, S. H. RELION: implementation of a Bayesian approach to cryo-EM structure determination. J. Struct. Biol. 180, 519-530 (2012).

77. Punjani, A., Rubinstein, J. L., Fleet, D. J. \& Brubaker, M. A. cryoSPARC: algorithms for rapid unsupervised cryo-EM structure determination. Nat. Methods 14, 290-296 (2017).

78. Pettersen, E. F. et al. UCSF Chimera-a visualization system for exploratory research and analysis. J. Comput. Chem. 25, 1605-1612 (2004).

79. Davis, I. W., Murray, L. W., Richardson, J. S. \& Richardson, D. C

MOLPROBITY: structure validation and all-atom contact analysis for nucleic acids and their complexes. Nucleic Acids Res. 32, W615-W619 (2004).
80. Barad, B. A. et al. EMRinger: side chain-directed model and map validation for 3D cryo-electron microscopy. Nat. Methods 12, 943-946 (2015).

81. Jorgensen, W. L., Chandrasekhar, J., Madura, J. D., Impey, R. W. \& Klein, M. L. Comparison of simple potential functions for simulating liquid water. J. Chem. Phys. 79, 926-935 (1983).

82. Georgiev, I. S. et al. Delineating antibody recognition in polyclonal sera from patterns of HIV-1 isolate neutralization. Science 340, 751-756 (2013).

83. Doria-Rose, N. A. et al. Mapping polyclonal HIV-1 antibody responses via next-generation neutralization fingerprinting. PLoS Pathog. 13, e1006148 (2017) 


\section{natureresearch}

Corresponding author(s): P.D. Kwong \& J.R. Mascola

Initial submission $\bigotimes$ Revised version

Final submission

\section{Life Sciences Reporting Summary}

Nature Research wishes to improve the reproducibility of the work that we publish. This form is intended for publication with all accepted life science papers and provides structure for consistency and transparency in reporting. Every life science submission will use this form; some list items might not apply to an individual manuscript, but all fields must be completed for clarity.

For further information on the points included in this form, see Reporting Life Sciences Research. For further information on Nature Research policies, including our data availability policy, see Authors \& Referees and the Editorial Policy Checklist.

\section{- Experimental design}

1. Sample size

Describe how sample size was determined.
208 HIV-1 strains were chosen to represent all major clades and circulating recombinant strains, and it is the largest panel that could be practically assessed by multiple antibodies.

58 of 208 strains ( $N=58$ ) with FP sequence "AVGIGAVF" were also used to assess animal plasma and monoclonal antibody neutralization. This FP sequence is most prevalent and matches the sequence of immunogen. This panel size allowed neutralization fingerprint assessment of immune plasma and comparison with antibodies.

Initial mouse experiments were carried out with group of 3 to allow the identification of positive responses. Group of at least 5 animals were chosen for subsequent mouse, guinea pig and rhesus macaque to have $97 \%$ confidence to detect $50 \%$ positive response rate, as defined by HIV-1 neutralization.

For structural determination by cryo-EM:

(a) for the VFP1.01-DS-SOSIP complex, 1255 exposure images were collected, which after manual masking, particle picking mean standard deviation stack filtering, 2D and 3D classification, yielded 14,931 particles for final 3D reconstruction and map refinement using 3-fold symmetry.

(b) for the VFP5.01-DS-SOSIP complex, 949 exposure images were collected, which after manual masking, particle picking mean standard deviation stack filtering, 2D and 3D classification, yielded multiple 3D classes, out of which the top 3 contained 7383, 4127 and 2099 particles, and were refined with C1 symmetry.

(c) for the VFP16.02-DS-SOSIP-VRC03-PGT122 complex, 1893 exposure images were collected, which after manual masking, particle picking mean standard deviation stack filtering, 2D and 3D classification, yielded 57,278 particles for final $3 \mathrm{D}$ reconstruction and map refinement using 3-fold symmetry.

(d) for the VFP20.01-DS-SOSIP-VRC03-PGT122 complex, 723 exposure images were collected (using energy filter), which after manual masking, particle picking mean standard deviation stack filtering, 2D and 3D classification, yielded 48,248 particles for final 3D reconstruction and map refinement using 3-fold symmetry.

No data were excluded from the analyses in this study.

All critical neutralization assays were reliably replicated as indicated in figures or methods.

All animal experiments were carried out in experimental groups. Although the magnitude varied, all of the experimental animals tested developed FP-directed antibody responses.

For vFP1-class antibodies, we describe several dozen. 


\section{Randomization}

Describe how samples/organisms/participants were allocated into experimental groups.
For animal study, allocation randomization was done by weight and age.

For crystallographic refinement, reflections chosen for free-R were chosen by standard methods.

For cryo-EM experiments, "gold-standard" 3D map refinement were carried out using two models, one for each half of the data. The two half maps had their phases randomized beyond resolutions at which unmasked FSC dropped below the FSC $=0.143$ criterion. A mask was applied to both half maps and an FSC calculated. This FSC was used along with the original FSC before phase randomization to compute the corrected FSC as reported in Chen, S. et al. Ultramicroscopy, 135, 24-35 (2013), and implemented within RELION and cryosparc.

\section{Blinding}

Describe whether the investigators were blinded to group allocation during data collection and/or analysis.
Investigators carrying out immunizations, ELISA assays, and neutralization assessment were blinded.

Note: all studies involving animals and/or human research participants must disclose whether blinding and randomization were used.

\section{Statistical parameters}

For all figures and tables that use statistical methods, confirm that the following items are present in relevant figure legends (or in the Methods section if additional space is needed).

n/a $\mid$ Confirmed

$\bigotimes$ The exact sample size $(n)$ for each experimental group/condition, given as a discrete number and unit of measurement (animals, litters, cultures, etc.)

A description of how samples were collected, noting whether measurements were taken from distinct samples or whether the same sample was measured repeatedly

$\searrow$ A statement indicating how many times each experiment was replicated

The statistical test(s) used and whether they are one- or two-sided (note: only common tests should be described solely by name; more complex techniques should be described in the Methods section)

A description of any assumptions or corrections, such as an adjustment for multiple comparisons

The test results (e.g. $P$ values) given as exact values whenever possible and with confidence intervals noted

A clear description of statistics including central tendency (e.g. median, mean) and variation (e.g. standard deviation, interquartile range) Clearly defined error bars

See the web collection on statistics for biologists for further resources and guidance.

\section{- Software}

Policy information about availability of computer code

\section{Software}

Describe the software used to analyze the data in this study.
All software are described in Methods section.

For crystal structures, diffraction data was processed with the HKL2000 suite. Structure solution was obtained by molecular replacement with Phaser. Refinement was carried out with Phenix. Model building was carried out with Coot. Structural figures were prepared with PyMOL.

For cryo-EM structures, data were acquired using Leginon. Motioncor2 was used for frame alignment and dose-weighting. CTF was estimated using the GCTF package. Particles were picked using DoG Picker within the Appion pipeline. 2D and 3D classifications were performed using RELION. After 3D classification in RELION, an additional step of ab initio reconstruction was performed using cryoSparc. 3D map refinements were carried out using either cryoSparc or RELION. Fits of HIV-1 trimer and Fab to the cryo-EM reconstructed maps were performed using Chimera. The coordinates were further fit to the electron density by an iterative process of manual fitting using Coot and real space refinement within Phenix. Molprobity and EMRinger were used to check geometry and evaluate structures at each iteration step. Figures were generated in UCSF Chimera and Pymol. Map-fitting cross 
correlations were calculated using Fit-in-Map feature in UCSF Chimera. Map-tomodel FSC curves were generated using EMAN2.

For MD simulation, a fully extended mannose 5 moiety at each $\mathrm{N}$-linked glycosylation sequon using our in-house software glycosylator. The fusion peptide structure was then grafted onto our full mannose 5 model followed by 5000 steps of conjugate gradient energy minimization in implicit solvent using NAMD. The obtained structure was then solvated in a $17 \AA$ padding water box, neutralized by the addition of $\mathrm{NaCl}$ at a concentration of $150 \mathrm{mM}$. The CHARMM force field was used for the parameterization of the protein (including CMAP corrections) and the mannose 9. TIP3P water parameterization 30 was used to describe the water molecules. Two independent molecular simulation were carried out using ACEMD molecular dynamics software on their METROCUBO workstation. Prody was used to perform principal component analysis of backbone atoms.

The associations between the sequence variability of FP neighboring glycosylation sites (HXB2 numbering 88, 241, 448 and 611) and the large panel neutralization using an in-house version of the approach implemented in $\mathrm{R}$ package SeqFeatR.

Prism7 was used to graph neutralization curves. Dendroscope 3 was used to visualize phylogenic tree of sequences, BioEdit v7.2.5 was used to visualize amio acid alignment, Octet software version 9.0 was used to analyze Octet binding result and generate binding curves.

For manuscripts utilizing custom algorithms or software that are central to the paper but not yet described in the published literature, software must be made available to editors and reviewers upon request. We strongly encourage code deposition in a community repository (e.g. GitHub). Nature Methods guidance for providing algorithms and software for publication provides further information on this topic.

\section{- Materials and reagents}

Policy information about availability of materials

8. Materials availability

Indicate whether there are restrictions on availability of unique materials or if these materials are only available for distribution by a for-profit company.

\section{Antibodies}

Describe the antibodies used and how they were validated for use in the system under study (i.e. assay and species).

\section{Eukaryotic cell lines}

a. State the source of each eukaryotic cell line used.

b. Describe the method of cell line authentication used.

c. Report whether the cell lines were tested for mycoplasma contamination.

d. If any of the cell lines used are listed in the database of commonly misidentified cell lines maintained by ICLAC, provide a scientific rationale for their use.
All described materials are available by MTA for non-profit research.

Previously published antibodies, including VRC34.01, Motavizumab, PGT151, CH07, ACS202, VRC01, 447-52D, PGT122, VRC03, VRC01-LS, 4E10, VRC07-523-LS and VRC07-G54W, were produced in the laboratory based on published sequences. None of these antibodies have an antibody validation profile; however the antigenic profile of these antibodies, assessed by ELISA, Octet and MSD, agrees with published descriptions as referenced throughout the manuscript.

Sheep D7324 antibody was purchased from AALTO Bio Reagents, with product code 7324 . The lyophilised antibody was reconstituted with $2 \mathrm{ml}$ of double distilled water before use, as instructed by the manufacture protocol. The serological activity of the antibodies is checked by ELISA

Expi293F (Invitrogen \#A14527); FreeStyle ${ }^{\text {TM }}$ 293-F Cells (Thermo Fisher catalog number Catalog number: R79007); HEK293S GnTI- (ATCC ${ }^{\circledR}$ CRL-3022 ${ }^{\mathrm{TM}}$ )

No, we didn't authenticate the commercial cell lines.

Yes, mycoplasma testing was performed through Hoechst DNA staining and tested to be negative.

No commonly misidentified cell lines were used. 
Policy information about studies involving animals; when reporting animal research, follow the ARRIVE guidelines

11. Description of research animals

Provide details on animals and/or animal-derived materials used in the study.

Female mice (C57BL/6) around 8 weeks old were purchased by GenScript from Yangzhou University, CN. Female Hartley guinea pigs with body weights of 300 grams were purchased from Charles River Laboratories, MA. Female and male Indian rhesus macaques with body weights of 2-9 kg were used for immunization studies. More details were included in the methods.

Policy information about studies involving human research participants

\section{Description of human research participants}

Describe the covariate-relevant population

characteristics of the human research participants. 